\title{
C-terminal Tail of $\beta$-Tubulin and its Role in the Alterations of Dynein Binding Mode
}

Kali A. Heale, Laleh Alisaraie ${ }^{*}$

School of Pharmacy, Memorial University of Newfoundland, 300 Prince Philip Dr., A1B 3V6, St. John's, Newfoundland, Canada

*Corresponding author

Email: laleh.alisaraie@mun.ca

Keywords: Microtubule Binding Domains of Dynein, $\beta$-CTT, Electrostatic Interactions, Molecular Dynamics Simulations, Principal Component Analysis, Normal Mode Analysis

Abstract: Dynein is a cytoskeletal molecular motor protein that moves along the microtubule (MT) and transports various cellular cargos during its movement. Using standard Molecular Dynamics (MD) simulation, Principle Component Analysis (PCA), and Normal Mode Analysis (NMA) methods, this investigation studied large-scale movements and local interactions of dynein's Microtubule Binding Domain (MTBD) when bound to tubulin heterodimer subunits. Examination of the interactions between the MTBD segments, and their adjustments in terms of intra- and intermolecular distances at the interfacial area with tubulin heterodimer, particularly at $\alpha-\mathrm{H} 16, \beta-\mathrm{H} 18$ and $\beta$-tubulin $\mathrm{C}$-terminal tail (CTT), was the main focus of this study. The specific intramolecular interactions, electrostatic forces and the salt-bridge residue pairs were shown to be the dominating factors in orchestrating movements of the MTBD and MT interfacial segments in the dynein's low-high affinity binding modes. Important interactions included $\beta$-Glu447 and $\beta$-Glu449 (CTT) with Arg3469 (MTBD-H6), Lys3472 (MTBD-H6-H7 loop) and Lys3479 (MTBD-H7); $\beta$-Glu449 with Lys3384 (MTBD-H8), Lys3386 and His3387 (MTBD-H1). The structural and precise position, orientation, and functional effects of the CTTs on the MT-MTBD, within reasonable cut-off distance for non-bonding interactions and under physiological conditions, are unavailable from the previous studies. The absence of the residues in the highly flexible MT-CTTs in the experimentally solved structures is perhaps in some cases due to insufficient data from density maps, but these segments are crucial in protein binding. The presented work contributes to the information useful for the MT-MTBD structure refinement. 


\section{Introduction}

The movement of dynein along microtubules (MTs) is an important means of transportation for large cellular cargoes within the cell [1].

Microtubules are linear cytoskeletal polymers composed of $\alpha / \beta$-tubulin heterodimers associating in a head-to-tail fashion. They play important roles in many cellular processes, such as cell division, motility, shape and cargo transport [2]. Due to their involvement in such biological processes, MTs have been investigated in anticancer drug discovery research [3-5]. The $\alpha$-tubulin is composed of 451 residues with sixteen $\alpha$-helices (H1 to H16) and thirteen $\beta$-strands (B1 to B13). $\alpha$-H15 (Val405 to Glu411), the C-terminal end of $\alpha$-H14 (Ile384 to LyS5S501) and the N-terminal end of $\alpha$-H16 (Glu415 to Val435) are located at the MT-MTBD interface.

$\beta$-tubulin is composed of 455 residues with eighteen $\alpha$-helices (H1 to H18) and sixteen $\beta$ strands (B1 to B16). $\beta-H 8$ (Met149-Glu160) and $\beta-H 9$ (Glu183-Asn197) are located at the MT-MTBD interface in the proximity of MTBD-H3. Both $\beta-\mathrm{H} 18$ and the $\beta$-tubulin $\mathrm{C}$-terminal tail (CTT, residues 438-455) are located near the MTBD-H1 at the interface of tubulin heterodimer and MTBD binding area, forming a heterotrimeric structure [6]. (Figure 1 and Figure S1)

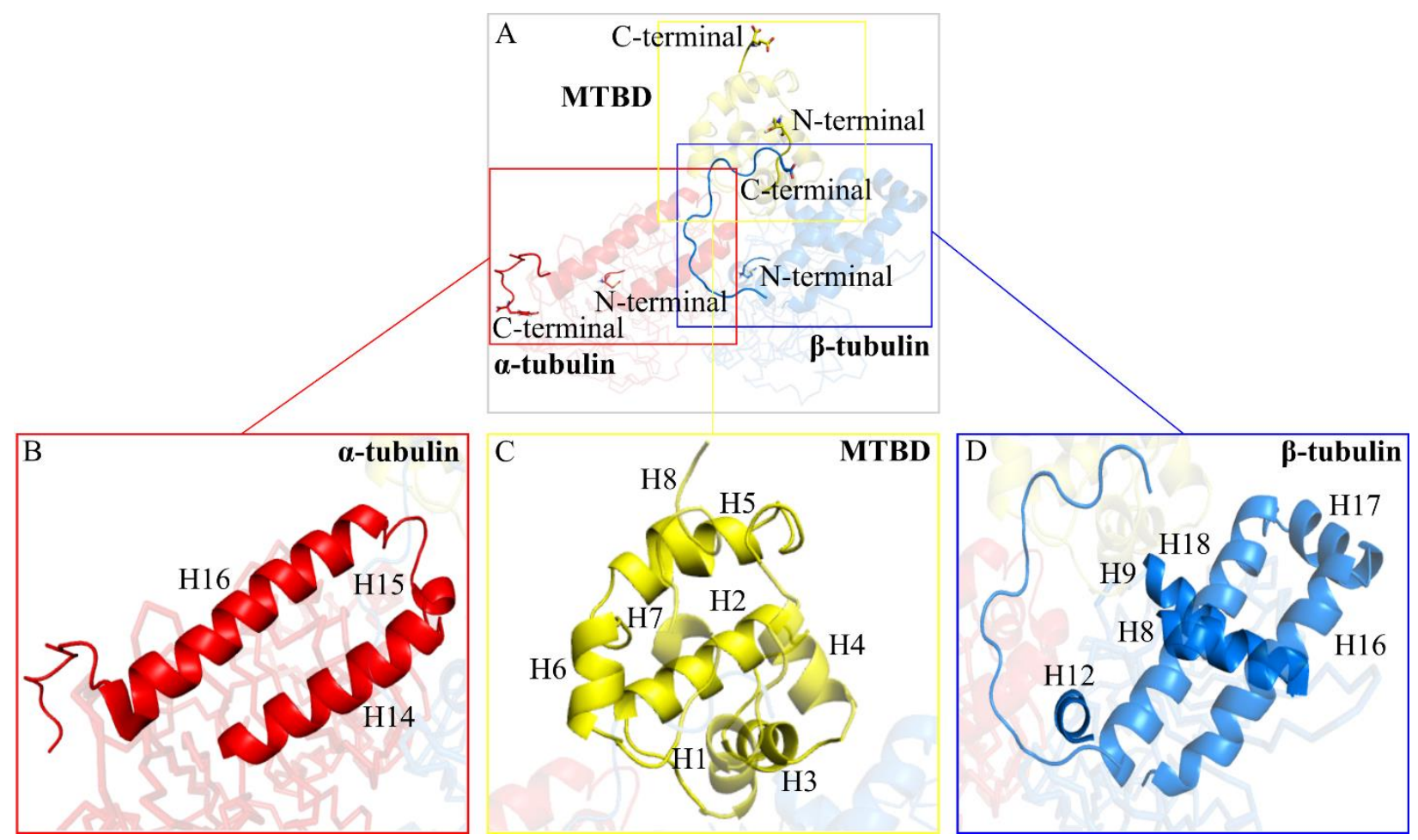

Figure 1: Overview of the heterotrimer, showing the MTBD (yellow) binding to the tubulin $\alpha / \beta$ - 
heterodimer (red and blue, respectively).

Molecular motors are enzymatically active biological molecules that utilize the chemical energy of ATP hydrolysis to perform essential mechanical work in a cellular system [7]. Outer arms dynein is referred to as a processive molecular motor at low concentration of ATP [8, 9]. Its movement along MTs is oriented in the direction of their minus end [7]. For wild type cytoplasmic dynein, this minusend directed movement occurs at a velocity of $1.21 \pm 0.02 \mu \mathrm{m} / \mathrm{s}[6]$. Dyneins are classified within the ATPase associated proteins with diverse cellular activities of $\mathrm{AAA}^{+}$superfamily [10]. They are responsible for the movement of cilia and also the transport of cellular organelles and other cargoes along MT [11]. Specifically, dynein allows the transport of cargoes above $2 \mathrm{MD}$, which are unable to move independently, given their high molecular weight. Cargoes include ribonucleoproteins, organelles and protein complexes [1]. In neurons, cytoplasmic dynein is responsible for the transport of such cargoes as TrKA, TrKB, BDNF in the cell axon and Rab5 endosomes, glycine receptor vesicles and mRNA protein complexes in the dendrites. This wide variety in cargo movement is permitted by variations in the conformation of light and light-intermediate chains as well as its complex formation with dynactin [12].

The MTBD is the site of MT binding [13]. The MTBD is a globular dynein subunit located at the tip of the coiled-coil dynein stalk between AAA4 and AAA5 [10]. Its structure comprises 107 residues and eight $\alpha$-helices (H1-H8).

Several structural components of dynein mediate the movement and binding of its MTBD to the MT. There are two separate registries that the dynein stalk has been shown to adopt upon binding to the MT. The $\beta+$ registry is adopted when the MTBD is not bound to the MT. In this conformation, MTBD-H1 is positioned perpendicular relative to the MT. The binding and subsequent switch to the high-affinity, $\alpha$-registry, causes a change in the position of MTBD-H1, bringing it more parallel relative to the MT central axis [13]. To reach this high-affinity state, the position of H1 is facilitated through greater interaction between dynein and the acidic segment of $\beta$-H12 [13]. It has been suggested that Asp417 and Glu421, from the acidic segment of $\beta$-H12, affect MT-MTBD binding [6].

One aim of the presented work was to investigate the conformational changes of the MTBD and identify the amino acids involved in the heterotrimer formation. This investigation also sought to determine the potential alterations of the secondary structure or folding of the segments at the MTMTBD binding site area, under physiological condition of $\mathrm{pH} 7.4$, when the system undergoes divergent modes of binding associated with the $\alpha$ - or $\beta$-registry. 
Molecular Dynamics (MD) simulations have been used to monitor and analyze the MT-MTBD movements and interactions as the MTBD transitions between various conformations. This study is different from previous work since it closely examined the interactions and binding affinities of the MTBD to determine both: the local events occurring at the atomistic level within the reasonable cutoff distances acceptable for non-bonding interactions and force field-defined ranges; as well as, the large scale collective motions of the domains affecting the formation of the low-to-high MTBD binding modes.

Valuable structural biology investigations, pertaining to the MT-MTBD system, have been published based on an $8.2 \AA$ resolution electron microscopic density map with an incomplete structure (i.e. missing residues) of the heterodimer tubulin without its CTTs [6], and other crystal structures of dynein, in the absence of the complete heterodimer tubulin $[13,14]$. In addition, a computational work that studied the number of potential contacts, excluding H-bonds, between MTBD and tubulin is available. The simulation was carried out within a shorter simulation time than of that in the presented work $(20$ ns, vs. 200 ns). The extra-wide cut-off distances employed there, ranged from $5 \AA$ up to $45 \AA$, consequently resulted in a great number of potential interactions [15]. Thus, it requires further experiments to confirm their actual occurrence in the physically acceptable ranges of non-bonding interactions.

The presented work takes further steps to elucidate the structural and functional roles of important segments, such as H1 (MTBD), $\beta$-H18 (MT-MTBD interface) and the $\beta$-CTT, which constantly fluctuate and act together with the MTBD. For instance, in the $8.2 \AA$-resolution electronmicroscopy (EMic) map of the system of study [6], the area in the proximity of the $\beta-\mathrm{H} 18$ was roughly filled by shifting the $\mathrm{N}$-terminal of the MTBD. Considering the resolution of the EMic structure, it suggests further structural refinement. This is particularly important in the $\beta-\mathrm{H} 18$ region, which determines MT-MTBD binding affinity. As such, this was given particular attention in the presented work. In addition, the CTTs of the tubulin heterodimer have not been solved in their published crystal structures (e.g., 1SA0 [16], 1JFF [17], etc.). It has also not been included in the EMic structure of the MT-MTBD [6]. The $\beta$-CTT consists of up to 34 residues and is constantly involved with the MTBD segments. Considering its absence in the experimentally solved structures, which possibly occurred due to the technical difficulties and high fluctuations of the CT'T loops, we built up and included the CTTs in the $\alpha$-, $\beta$-tubulin structure. The inclusion of these CTTs and analyses of their function expand our perspective in viewing the potential effects of CTTs post-translational modifications on the MTdynein binding. 


\section{Materials and Methods}

The structure of the MT-MTBD complex was retrieved from the Protein Data Bank (PDB) [www.pdb.org.] under the 3J6P [6] PDB code. The structure was obtained from EMic with a resolution of $8.2 \AA$. The tubulin heterodimer structure was based on the Sus scrofa sequence, while the cytoplasmic dynein heavy chain was from Dictyostelium discoideum.

Residues 1, 35-60, and 440-451 (C-terminal) of $\alpha$-tubulin as well as residues 1, and 438-455 (Cterminal) of $\beta$-tubulin were missing in the EMic structure [6]. The C-termini segments have remained unsolved in the crystal structures of the tubulin monomers $[16,17]$ due to their high flexibilities; thus, in this work, those segments were first constructed according to the sequence data from Uniprot with accession codes P02550 for $\alpha$-tubulin (chain A) and P02554 for $\beta$-tubulin (chain B). The constructed segments were added to the tubulin amino acids sequence in 3J6P [6] to complete the monomers structures for the simulation study, as it is a common method in the in silico study of protein structures $[3,4,18]$. The constructed segments formed a simple coil shape, which could be also predicted by the Phyre, protein folding predictor tool and search engine [19], for the missing segments.

To compare the results of the in silico simulations in this work, and in vitro experiments on different species $[6,13]$, sequence alignment was performed using the highly accurate alignment algorithm of the Clustal W program [20,21]. The program was used to compare the amino acid sequences of $D$. discoideum and M. musculus cytoplasmic dynein heavy chain (UniProt accession codes P34036 [22] and Q9JHU4 respectively); and sequences of S. scrofa $\alpha$ - and $\beta$-tubulin (UniProt accession codes P02550 [23] and P02554 [24] respectively) and S. cerevisiae $\alpha$ - and $\beta$-tubulin (UniProt codes P09733 [25] and P02557 [26], respectively). Discoideum and M. musculus cytoplasmic dynein heavy chain amino acid sequences were found to have 51.4\% identity (55 identical amino acids out of 107). Helices H6 and H7 were highly conserved between the species. (Figure 1 and Figure S2)

The percent of amino acid identity between S. scrofa and S. cerevisiae $\alpha$-tubulin was found to be 73.8\% (333 identical amino acids out of 451 total amino acids). H1 (Gln11-His28) is highly conserved between the species; only one amino acid of the 17 total amino acids, Val14, was not identical. A stretch of the sequence, which encompassed eight of the amino acids of the $\alpha$-H14 C-terminal, (Lys394 to Lys401), $\alpha-\mathrm{H} 15$ (Val405 to Glu411), and five amino acids from the $\alpha$-H16 N-terminal (Glu415 to Phe418) was highly conserved between the two species. The $\alpha$-H15, the C-terminal of $\alpha-\mathrm{H} 14$ and the $\mathrm{N}$-terminal of $\alpha-\mathrm{H} 16$ were present at the MT-MTBD interface.

The $\alpha-\mathrm{H} 11$ was conserved (Ala289 and Glu290 to Phe295), with only Val288 not identical between the species. For the $S$. scrofa and $S$. cerevisiae $\beta$-tubulin chain comparison, the percent sequence identity 
was found to be $73.2 \%$ (333 identical amino acids out of 455 amino acids). Several identical sequences can be seen, namely, $\beta-\mathrm{H} 8$ and $\beta-\mathrm{H} 18$, which were both present at the MT-MTBD interface. $\beta-\mathrm{H} 17$ was partially conserved with only one amino acid differing between the species (Gly410). (Figure 1 and Figure S2)

The secondary structure coding, which was used throughout this text, was based on the 3J6P structure (Uchimura et al. [6]).

Molecular Dynamics (MD) simulations were used to study and analyze the protein subunits' movement and conformational changes, utilizing the software package GROningen MAchine for Chemical Simulations (Gromacs) v. 2016.3 based on GROMOS 54A7 force field [27, 28] to generate topology data for the atoms in the system of study [6]. Gromacs uses classical mechanics to model the movement of a group of atoms [29, 30]. The program solves the Newtonian Equation of motion for all atoms in a molecular system [31]. The MTBD-tubulin heterodimer system was solvated in a water box, using the SPC water model and 43 sodium cations $\left(\mathrm{Na}^{+}\right)$were added to neutralize the net charge of the system. The proteins were centred in a cubic box with a distance of $1.0 \mathrm{~nm}$ from the edge of the box with a total volume of $1981.3 \mathrm{~nm}^{3}$.

Energy Minimisation (EMin) was performed using the steepest descent (SD) algorithm [32] to remove atomic clashes and explore the conformation of the structure near the global minimum. Given the initial coordinates of atoms obtained from cryo-EMic data for the MT-MTBD molecular system [6], the forces on the atoms of the heterotrimeric system were calculated for both bonded and nonbonded interactions [31].

Periodic Boundary Conditions (PBC) were applied to minimize edge effects. Within the PBC, the box of water containing the solvated system was surrounded by translated versions of this box. As there were many copies of each molecule, Gromacs employed the minimum image convention to evaluate non-bonded interactions such that only one image of an atom was used in calculating interactions [31]. Thus, all non-bonded interactions calculated were within half of the length of the shortest box vector [31]. For longer-range interactions, Particle Mesh Ewald (PME), a lattice sum method, was used for calculations [33].

In the NVT or position restraint (PR) phase of the MD simulations, the md integrator was used to integrate Newton Equation of Motion for a 500 ps simulation with step size of 0.002 ps. The LINear Constraint Solver (LINCS) algorithm [34] was employed to restrain all bonds. In the PR, the velocity rescaling thermostat was used to relax the temperature of the system to a reference temperature of $300 \mathrm{~K}$ [35]. The velocity rescaling thermostat is a modified version of the Berendsen 
thermostat, providing an exponential movement of the system temperature to the reference temperature while still allowing the generation of a proper canonical ensemble through the consideration of kinetic energy fluctuations [31]. The $\mathrm{md}$ integrator was used in the NPT or Equilibration (EQ) phase of the MD simulation, with a step-size of 0.002 ps and a duration of $10 \mathrm{~ns}$.

The compressibility was set to $4.5 \times 10^{-5}$ bar. A constant pressure of 1.0 bar was reached through the Berendsen pressure-coupling scheme. For the data collection phase of the MD simulations, the md integrator was again used, and the calculations were performed for $200 \mathrm{~ns}$ with 0.002 ps step size. After the convergence at $\sim 50 \mathrm{~ns}$, analysis was performed on the $50 \mathrm{~ns}-200 \mathrm{~ns}$ segment of the production MD trajectory. (Figure S1)

GROMACS 2016 double-precision was used for the Normal Mode Analysis (NMA) [36, 37] to observe tubulin and MTBD's large scale movements and their dynamic deformations. The eigenvector values of a Hessian matrix were calculated following a thorough minimization of the heterotrimeric structure using the leap-frog algorithm, coulomb and van der Waals modifiers with the Verlet list scheme. An ensemble of conformations was generated and scaled to the Cartesian coordinates for data analysis with a mass-weighted process. For Essential Dynamics (ED) [38, 39], also known as Principle Component Analysis (PCA), a trajectory matrix was first generated by removing major translational and rotational motions of the protein and fitting it to the EMin structure. This was used as the reference structure for the coordinates of the protein's atoms. The matrix was then used to generate a symmetric covariance matrix for its diagonalization. Transforming the MD trajectory matrix to the new orthogonal matrix also consisted of eigenvectors sorted in descending order of their eigenvalues, which were also used for screening and filtering the MD trajectory and visual inspections of its low-frequency conformations. MD simulations were performed on the Compute Canada HighPerformance Computer Clusters (HPC), including ACENET, WestGrid and Graham.

\section{Results and Discussion}

\subsection{Local displacements and interactions}

The starting structure for this investigation was 3J6P [6], an $8.2 \AA$ EMic solved structure, in which the MTBD was positioned on the dorsal side of the tubulin dimer, at the interface between $\alpha$ and $\beta$-tubulin. MTBD-H1,-H3, and -H6 were the major helices at the MT-MTBD interface. In the initial conformation, MTBD-H1 (Lys3385 to Ser3393), was positioned perpendicular relative to the tubulin dimer and in close proximity to the intra-dimer interface. H3 was located on the side of the 
MTBD closest to $\beta$-tubulin and in the proximity of $\beta-\mathrm{H} 8$ and $\beta-\mathrm{H}$. MTBD-H6 was located on the side of the MTBD closest to $\alpha$-tubulin and was in close proximity to $\alpha-\mathrm{H} 16$ [6]. (Figure 1)

The system converged at approximately $50 \mathrm{ns,} \mathrm{and} \mathrm{accordingly,} \mathrm{all} \mathrm{data} \mathrm{analysis} \mathrm{was} \mathrm{conducted} \mathrm{after}$ this time point. (Figure 2A)
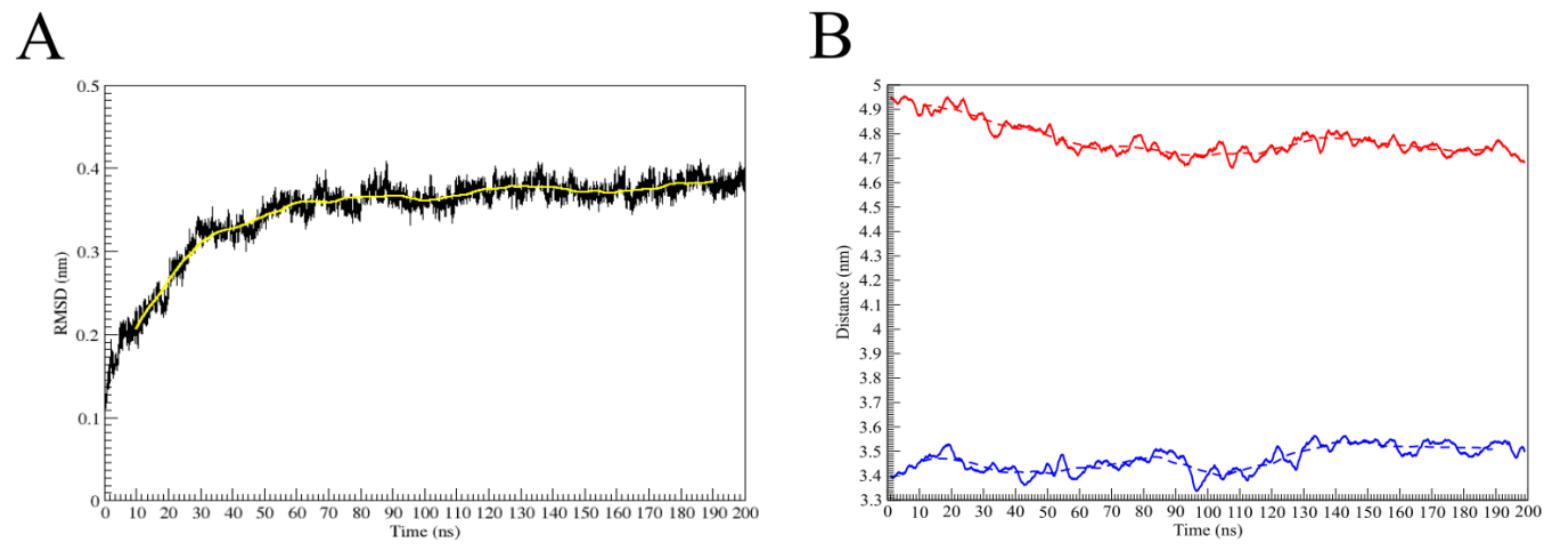

Figure 2: (A) RMSD data for the heterotrimer throughout the 200 ns simulation and (B) dCOM between MTBD and $\alpha$-tubulin (red) and MTBD and $\beta$-tubulin (blue) showing 100-point average trend line (solid lines) and 1000-point average (dashed lines).

To assess the local displacement of the MTBD segments according to its conformational changes, the distance from Center of Mass (dCOM) of the entire MTBD structure was calculated relative to both $\alpha$ - and $\beta$-tubulin. (Figure 2B)

Results indicated that the $\mathrm{dCOM}$ between the MTBD and $\alpha$-tubulin decreased throughout the $200 \mathrm{~ns}$, from $49.5 \AA(\sim 1 \mathrm{~ns})$ to the convergence frame with $48.3 \AA(\sim 50 \mathrm{~ns})$, and the final magnitude of $46.8 \AA(\sim 199$ ns) that was less than the dCOM between the MTBD and $\alpha$-tubulin in the EMin structure, (48.5 $\AA$ ), suggesting that the overall interaction between the MTBD and $\alpha$-tubulin segments at the interface of the two domains became stronger over the course of the simulation. (Figure 2B) Conversely, the $\mathrm{dCOM}$ between the MTBD and $\beta$-tubulin generally tended towards an increase over the $200 \mathrm{~ns}$, from the initial dCOM of $34.0 \AA$ ( 1 ns) to the convergence frame with $34.5 \AA$ (at $\sim 50$ ns) and the final distance of $35.0 \AA(\sim 200 \mathrm{~ns})$, indicating a general increase in the distance between the center of mass of the MTBD and $\beta$-tubulin, particularly following $\sim 97$ ns. (Figure 2B) 


\section{MTBD-H1}

Given that MTBD-H1 (Lys3385-Ser3393) was located at the MT-MTBD interface, its interactions with the MT was closely examined. Residues comprising H1 (Lys3385 to Ser3393) had an average RMSF value of $\sim 1.3 \AA$ across the entire helix, with the greatest RMSF (1.4 $\AA$ ) observed at Lys3385, and the low est average RMSF value (1.2 ^) observed at Glu3390. (Figure 3)

A

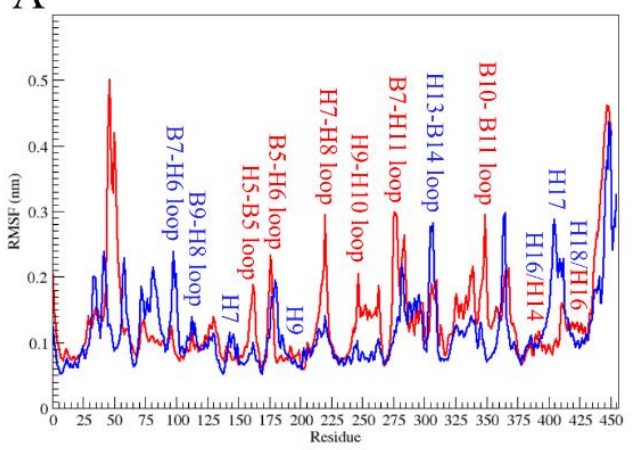

$\mathrm{C}$
B

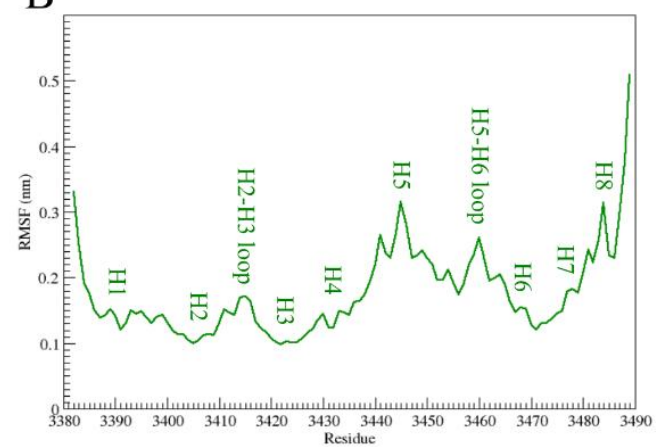

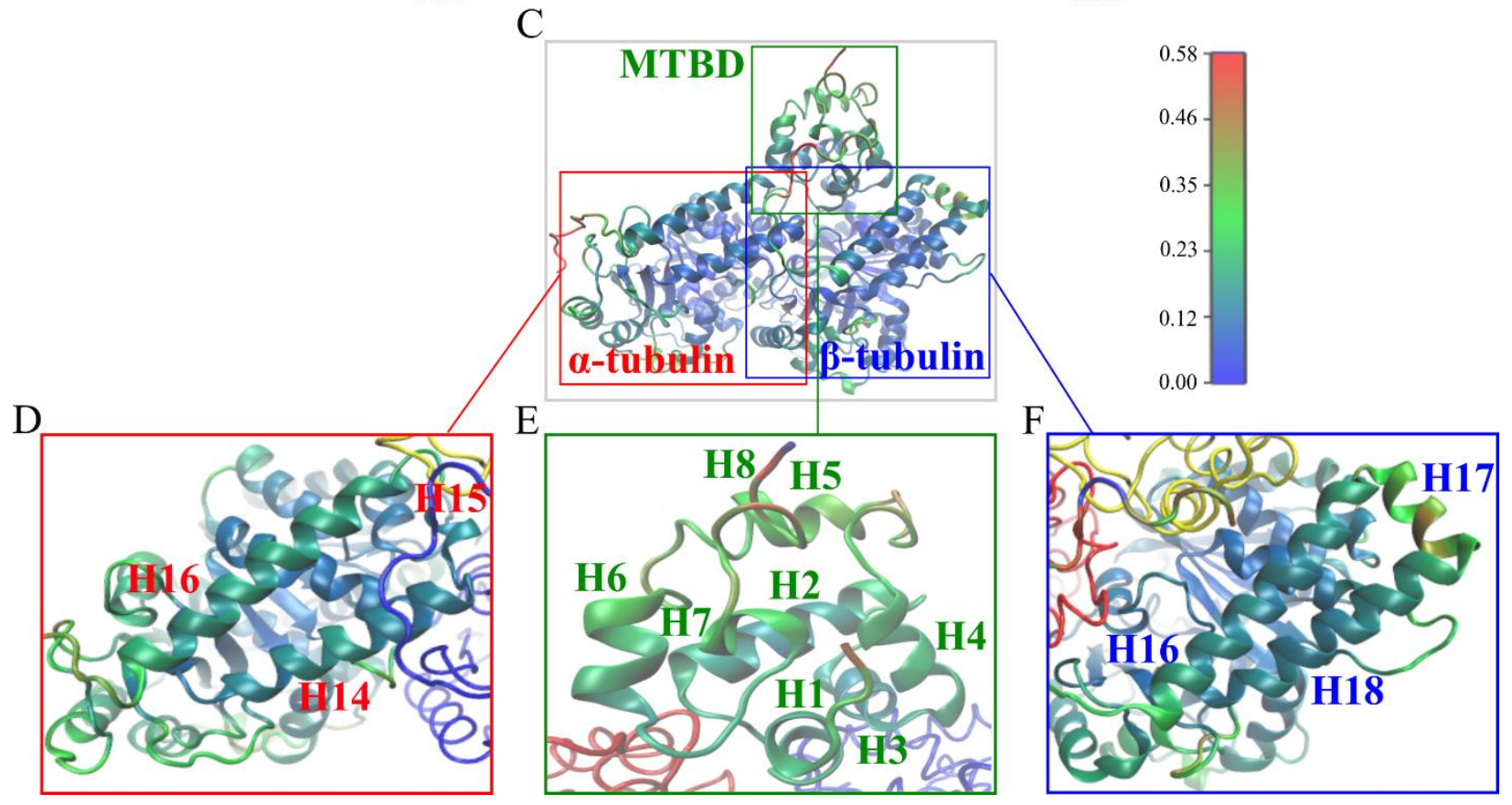

Figure 3: Average RMSF values for each residue of (A) $\alpha$-tubulin (red), $\beta$-tubulin (blue) and (B) dynein MTBD after convergence (i.e. 50 ns to 200 ns). (C) Heterotrimeric structure colored in accordance with RMSF values showing (D) $\alpha$-tubulin, (E) MTBD, and (F) $\beta$-tubulin.

The percent of helicity of MTBD-H1 ranged between 58.7\% (Leu3388), and 81.1\% (Asp3389).

\section{(Figure S5)}

The MTBD-H1, -H3 and -H6 have been shown to displace the greatest distance upon the 
conformational change from low- to high-affinity. During this conformational change, the repositioning of $\mathrm{H} 1$ and $\mathrm{H} 3$ is thought to be critical [13]. Following convergence at $50 \mathrm{~ns}$, the dCOM between the $\mathrm{H} 1$ and $\mathrm{H} 3$ mainly increased, up to $\sim 15.0 \AA$. The average dCOM between MTBD-H1 and $-\mathrm{H} 3$ was $\sim 13.2 \AA$, while the dCOM of the EMin structure was $12.6 \AA$. In fact, the dCOM of the MD structure remained higher than that of the EMin structure during the full 150 ns following convergence. (Figure S6A)

A similar trend was observed between the two $\mathrm{N}$-terminal residues of $\mathrm{H} 1$ and $\mathrm{H} 3$. The distance between Lys3385 (N-terminal residue of H1) and Trp3419 (N-terminal residue of H3) was relatively high (up to $23.1 \AA$ ). In this case, the dCOM between Lys3385 and Trp3419 was also greater than that of the EMin structure $(16.9 \AA)$ for the entirety of the simulation following convergence. (Figure S6B) Given this information, the effect of MTBD-H1-H3 distance was examined in relation to the conformation of the MTBD. The relative positions of $\mathrm{H} 1$ and $\mathrm{H} 3$ were examined at the minimum and maximum distance following convergence. (Figure 4)
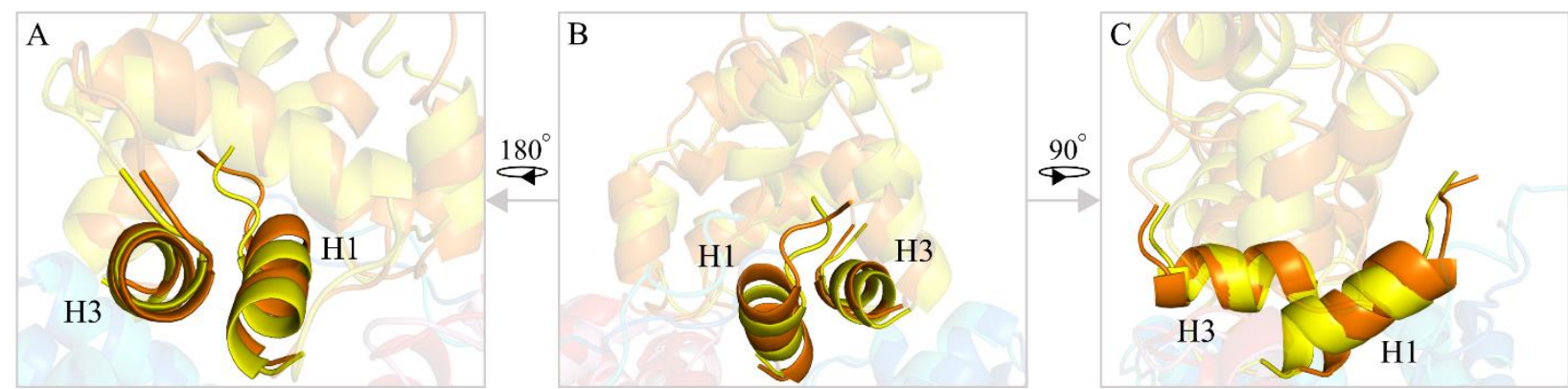

Figure 4: Comparison between the positions of MTBD-H1 and H3 at minimum (79.0 ns, yellow) and maximum (145.0 ns, orange) distances from one another showing (A) $180^{\circ}$ rotation from "Main" view, (B) "Main" view, showing MTBD N-terminal and (C) $90^{\circ}$ counter clockwise rotation from "Main” View.

As the MD progresses, the system becomes progressively stable, which accounts for the structural switch into a more stabilized form of the low-affinity conformation. (Figure 4) In the low-affinity conformation of the MTBD, H1 is proposed to be positioned perpendicular between the tubulin dimer. How ever, upon a switch to the high-affinity conformation, H1 is thought to swing upward to a final position above $\mathrm{H} 3$, becoming increasingly parallel relative to the MT. Regarding the position of $\mathrm{H} 3$ in the low-affinity conformation, this helix is located above and behind H1. During the switch from the low - to high-affinity conformation, H3 is proposed to move forward 
and downward to its final position closer to the $\beta$-tubulin [13], supporting this, the PCA result has exhibited a more pronounced collective movement of H1 vs. H3. (Figure 9)

In terms of the movement of $\mathrm{H} 1$ relative to $\alpha-\mathrm{H} 14$, the data suggested the distance between them generally decreased during the MD trajectory. (Figure S6C)

The proximity of MTBD-H1 and $\alpha-\mathrm{H} 14$ compared to that in the EMin structure showed MTBD-H1 was shifted farther away from the rest of the heterotrimer such that MTBD-H1 $(\mathrm{N}$ terminal) was in close proximity to the $\beta$-CTT. Relative to the EMin structure, $\alpha-\mathrm{H} 14$ was shifted in the direction of $\beta$-tubulin. (Figure $5 \mathbf{A}$ and $\mathbf{5 B}$ )

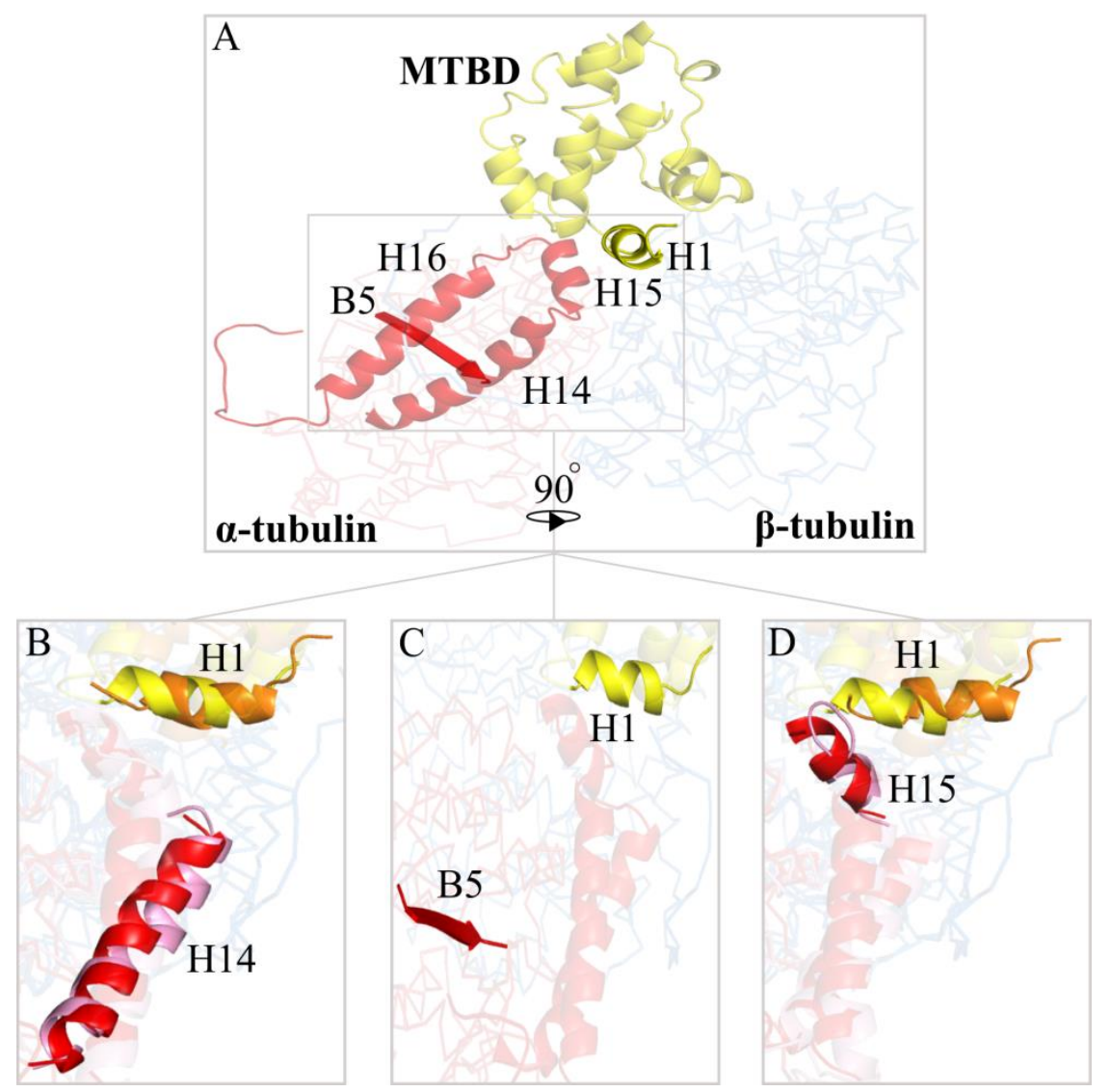

Figure 5: (A) cryo-EMic heterotrimer structure with $\alpha$-tubulin (red), $\beta$-tubulin (blue) and MTBD (yellow) (B) EMin heterotrimer superimposed with structure at 60 ns showing MTBD-H1 (orange) and $\alpha-\mathrm{H} 14$ (pink), (C) EMin heterotrimer showing the position MTBD-H1 relative to $\alpha$-B5, and (D) EMin structure superimposed with structure at 144 ns showing MTBD-H1 (orange) and $\alpha$-H15 (pink).

MTBD-H1 and $\alpha-\mathrm{H} 15$

The average RMSF values for $\alpha$-H15- a helix located at the MT-MTBD interface (Val405-Glu411) ranged from $7.5 \AA$ to $9.0 \AA$. (Figure 3) 
The data provides evidence that MTBD-H1 became farther aw ay from $\alpha-\mathrm{H} 15$ over the course of the MD simulation, since there was a substantial shift of $\mathrm{H} 1$ away from the rest of the heterotrimer, especially at $\sim 144 \mathrm{~ns}$, where the distance between MTBD-H1 and $\alpha-\mathrm{H} 15$ was highest, and the MTBD $\mathrm{N}$-terminal became closer to the $\beta$-CTT. (Figure 5A \& 5D and Figure S6D)

\section{MTBD-H1 and $\alpha$-B5 strand}

The distance of MTBD-H1 was measured against a stable reference point, $\alpha$-B5 strand, due to its placement within the interior of the $\alpha$-tubulin molecule and its relatively fixed coordination throughout the MD trajectory. The distance between $\mathrm{H} 1$ and $\alpha$-B5 generally increased throughout the trajectory. Following convergence, the $\mathrm{dCOM}$ between $\mathrm{H} 1$ and $\alpha-\mathrm{B} 5$ was greater than that of the EMin structure $(37.6 \AA)$. (Figure $\mathbf{5}$ and Figure 6A)

Given the relative movement of these segments, residue interactions were examined across the MTMTBD interface. This included both hydrogen bonding and salt bridge interactions. Ser3393, Thr3399, Ser3471, and Arg3469 from the MTBD formed hydrogen bonds to Pro263, Glu414, Glu415, and Gly416 of tubulin heterodimer. (Table 1 and Table S1)

\begin{tabular}{cccc}
\hline Donor residue & Chain/helix & Acceptor residue & Chain/helix \\
\hline Ser3393 & MTBD-H1 & Pro263 & $\beta$-H12-B10 loop \\
Thr3399 & MTBD-H2 & Glu414 & $\alpha$-H15-H16 loop \\
Ser3471 & MTBD-H6-H7 loop & Glu415 & $\alpha-H 16$ \\
Gy416 & $\alpha-H 16$ & Arg3469 & MTBD-H6 \\
\hline
\end{tabular}

Table 1: Hydrogen bonds determined across the MT-MTBD interface.

The distance between pairs of the hydrogen bond-forming residues was analyzed. For instance, the distance between Ser3393 (MTBD-H1) and $\beta$-Pro263 ( $\beta$-H12-B10) decreased from $7.3 \AA$ to $5.3 \AA$, indicating that they became closer in space as the 200 ns progressed. (Figure S6E)

The displacement of $\mathrm{H} 1$ was further examined when salt bridges were evaluated across the MTMTBD interface. In the detection of salt bridges, a more conservative cut-off value of $3.2 \AA$ was used to identify pairs of interacting residues. A salt bridge was detected between Glu3390 (MTBD-H1) and $\alpha$-Arg402 ( $\alpha$-H14-H15 loop). (Table S1, Figure S6R) 
A

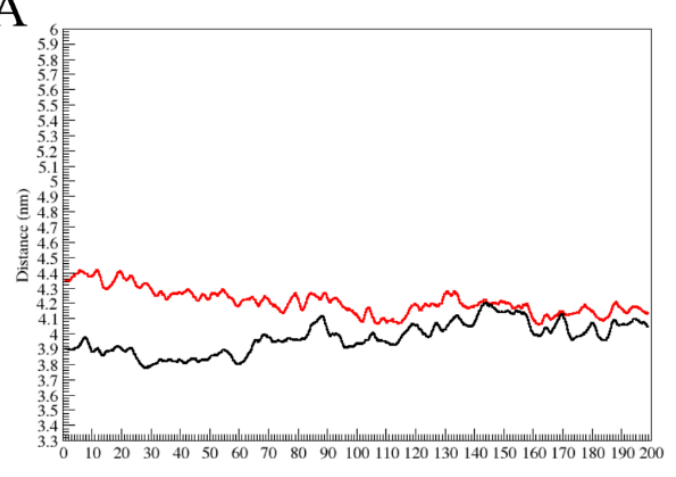

$\mathrm{C}$

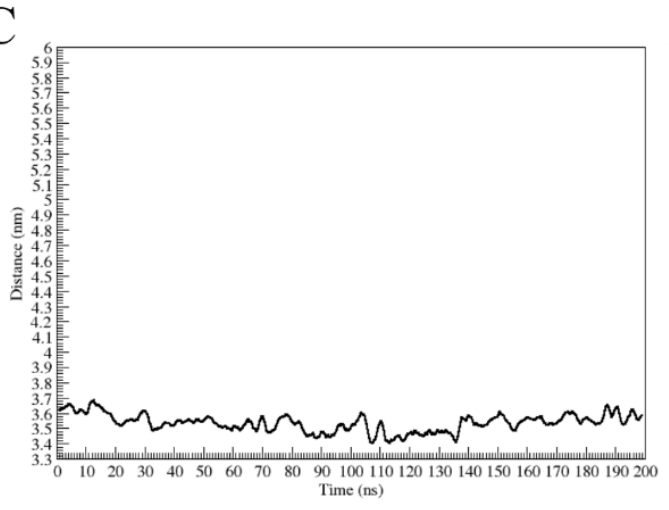

B

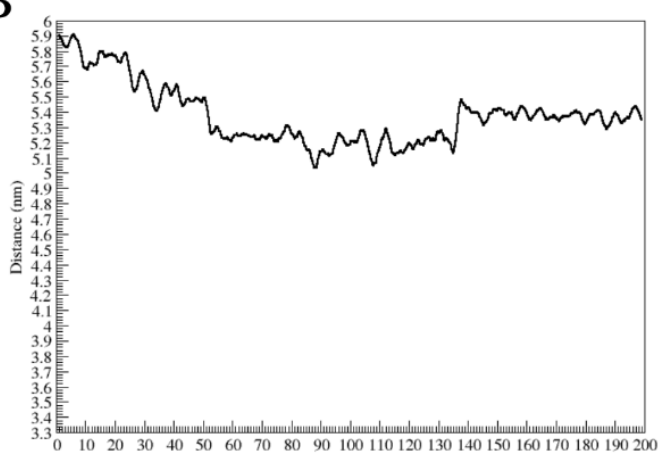

$\mathrm{D}$

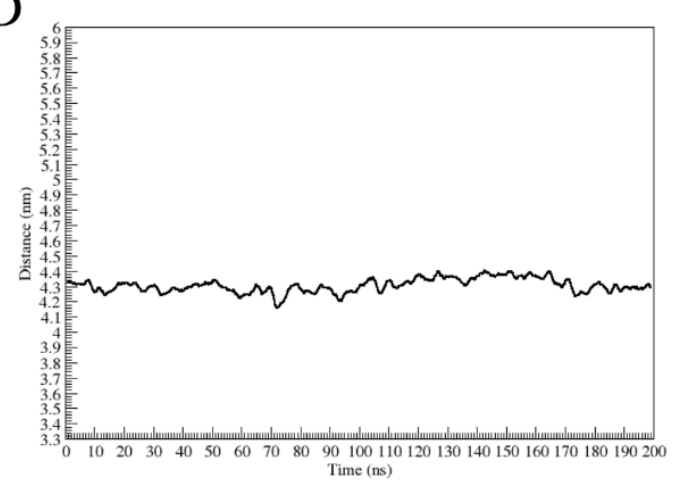

Figure 6: dCOM values between $\alpha-\mathrm{B} 5$ and MTBD (A) H1 (black) and $\mathrm{H} 3$ (red), (B) H5, (C) H6 and (D) $\mathrm{H} 7$.

Salt-bridge interaction is thought to play an important role in allowing the registry switch that facilitates ATPase activation and CC1-CC2 sliding during the conformational change of the MTBD [6]. Another salt bridge interaction was detected between Lys3396 (MTBD-H1-H2 loop) and Glu411 ( $\alpha-\mathrm{H} 15)$. The dCOM between Lys3396 and $\alpha$-Glu411 fluctuated and tended towards an increase. This indicated that this portion of the H1-H2 loop was moving farther aw ay from $\alpha$-Glu411. It should be noted that this interaction was one of only four salt bridges observed between the MTBD and $\alpha$-tubulin. (Figure S6F, Table S1 and Supplementary Information)

However, one potential salt-bridge interaction from within the $\alpha$-subunit was examined. The dCOM between $\alpha$-Glu415 and $\alpha$-Arg402 was plotted over the 200 ns trajectory. (Figure S6U)

MTBD-H1 and $\beta$-H18 through $\beta$-CTT

The distance between $\beta-\mathrm{H} 18$ and the MTBD increased after convergence. (Figure S6A)

The results indicated that the CT'T and the MTBD underwent many periods of close proximity during 
their trajectory, which was of interest to this investigation. (Figure S6B)

The distance between the $\beta$-CTT and MTBD-H1 was easily discernible (17.5 $\AA$ to $14.7 \AA$ ). (Figure S6D)

Over half of the residues in the tubulin heterodimer that were shown to form salt bridges were located in either $\beta$-H18 or the $\beta$-CT'T. For each of these residues, at least one interaction was formed with MTBD-H1. (Table S1)

The investigation highlighted three particular residues from $\beta-\mathrm{H} 18$, which interacted with $\mathrm{H} 1$; these included Glu420, Asp431 and Glu431. $\beta$-Glu447 ( $\beta$-CTT) formed salt-bridges with Lys3384 (MTBDN-terminal), Lys3386 (MTBD-H1) and His3387 (MTBD-H1), while $\beta$-Glu449 ( $\beta$-CT'T) formed saltbridges with Lys3384 (MTBD N-terminal), Lys3386 (MTBD-H1), and His3387 (MTBD-H1). (Table

\section{S1, Figure S6G and Figure S6H)}

In terms of hydrogen bonding, between three and twelve bonds were detected between the MTBD and $\beta$-tubulin over the trajectory. More specifically, the number of hydrogen bonds between the MTBD and $\beta-\mathrm{H} 18$ fluctuated between zero and three while the number of bonds between the MTBD and the $\beta$-CTT fluctuated between zero and four. (Figure S4)

\section{MTBD-H2}

MTBD-H2 and $\alpha-\mathrm{H} 15$

For $\alpha$-tubulin, the main helix at the MT-MTBD interface was $\alpha$-H15 (Val405 to Glu411). The minimum percent helicity of $\alpha-\mathrm{H} 15$ was $28.0 \%$ (Glu411), while the maximum percent helicity was 80.6\% (His406). This helix showed two distinct levels of helicity. The first three residues of the helix, (Val405 to Trp407), had an average percent helicity of $71.4 \%$ while the final four residues (Tyr408 to Glu411) had an average percent helicity of $25.5 \%$. The final four residues, which were unfolded for a greater duration of time than the first three residues, were oriented closer to MTBD-H2. (Figure S5) The data indicated that $\mathrm{H} 2$ displaced distance tow ards $\alpha-\mathrm{H} 15$ as the simulation progressed. (Figure S6I)

The terminal segment of the $\alpha$-H15 (i.e. also the $\mathrm{C}$-terminal) had a lower percent helicity than the rest of the helix. Relative to the EMin structure, the N-terminal end of MTBD-H2 shifted closer towards tubulin (at 145 ns), bringing it near $\alpha-\mathrm{H} 15$. (Figure 7)

In terms of salt bridge formation, $\alpha-$ Glu411 ( $\alpha$-H15) interacted with Lys3402 (MTBD-H2), in addition to Lys3396 (H1-H2 loop) as previously discussed. The distance between $\alpha$-Glu411 and Lys3402 fluctuated. Given the initial dCOM of these two residues, they tended to displace distance closer to 
one another throughout the trajectory. (Figure S6F, Table S1 and Supplementary Information)

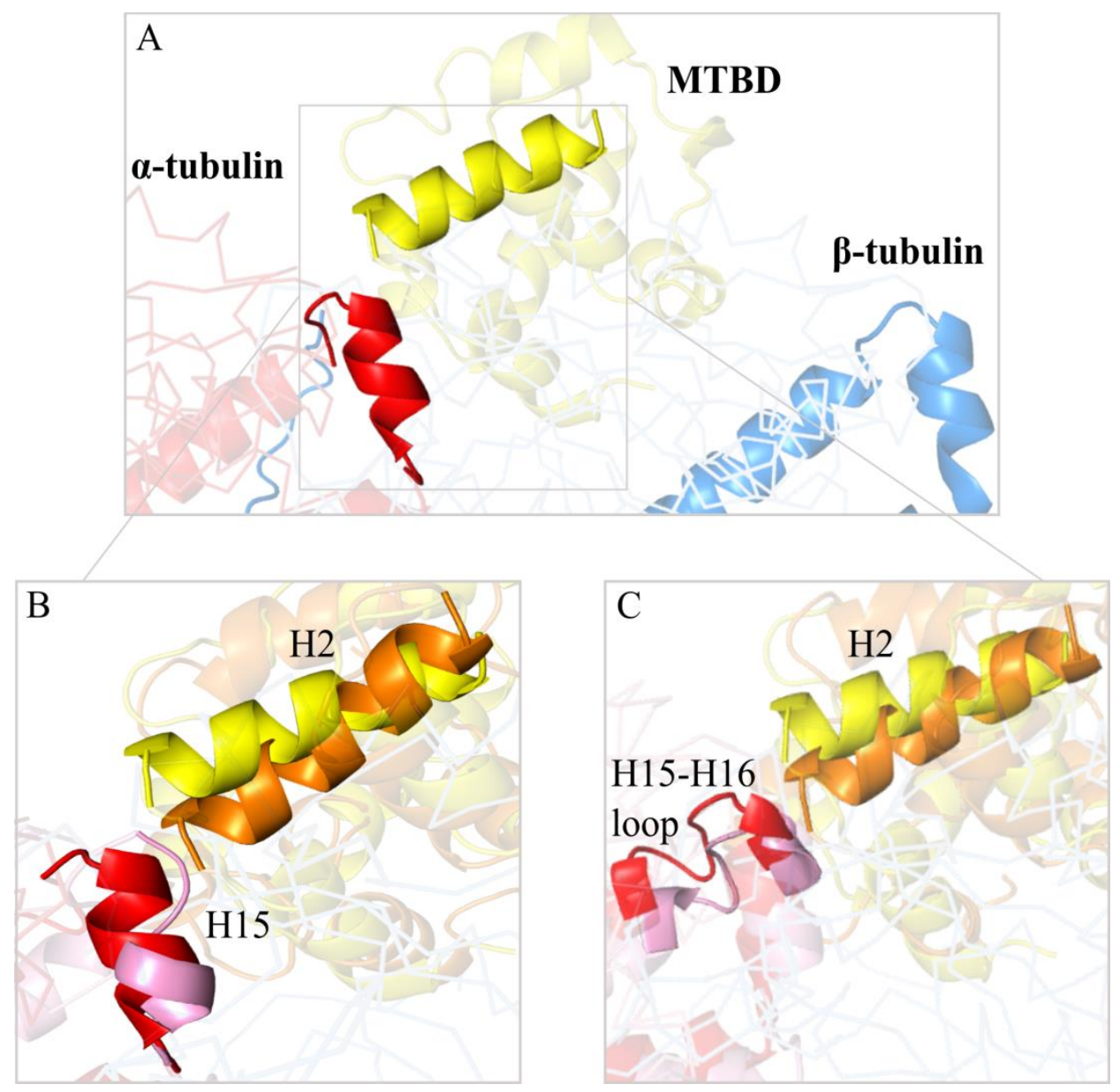

Figure 7: (A) EMin structure of MTBD-H2 (yellow), $\alpha-\mathrm{H} 15$ and $\alpha-\mathrm{H} 15-\mathrm{H} 16$ loop (red) and $\beta$-tubulin (blue), (B) EMin structure superimposed with MTBD-H2 (orange) and $\alpha$-H15 (pink) structure at $145 \mathrm{~ns}$, and (C) EMin structure superimposed with MTBD-H2 (orange) and $\alpha-\mathrm{H} 15-\mathrm{H} 16$ loop (pink) at $87 \mathrm{~ns}$.

MTBD-H2 and $\alpha-\mathrm{H} 15-\mathrm{H} 16$ loop

Thr3399 (MTBD-H2) was found to hydrogen bond with $\alpha$-Glu414 ( $\alpha$-H15-H16 loop). When the distance was examined between these two residues, a clear progression towards a decreasing dCOM was observable. The data highlighted local displacement of the MTBD-H2 in the direction of $\alpha-\mathrm{H} 15$ and the $\alpha-\mathrm{H} 15-\mathrm{H} 16$ loop. The examination even found a salt bridge interaction between MTBD-H2 and $\alpha$-Glu411 ( $\alpha$-H15); this is significant given that very few other salt bridges formed between the MTBD and $\alpha$-tubulin. (Figure 7) 


\section{MTBD-H3}

For H3 (Trp3419-Ile3426), the average RMSF over the entire helix was $\sim 1.1 \AA$. In terms of H3 helicity, a minimum percent helicity of $35.4 \%$ was observed at Ile3426, while a maximum percent helicity of $88.3 \%$ was observed at Ile3422. Both residues were located on the same side of MTBD-H3 in the proximity of MTBD-H2. (Figure 3 and Figure S5)

MTBD-H3 and $\beta-\mathrm{H} 8$

The minimum percent helicity of $\beta-\mathrm{H} 8$ was $63.9 \%$, occurring at Ser155, while the maximum percent helicity was $85.6 \%$ at Ile154. (Figure S5)

These neighbouring residues were both located in the middle of the $\beta-\mathrm{H} 8$ amino acid sequence, and based on their positioning, were very unlikely to interact with any residues other than those of $\beta$ tubulin. Hence any disengagement from helix formation would be unlikely to play a significant effect on the MTBD binding affinity. Following convergence, the dCOM values between the MTBD-H3 and $\beta-\mathrm{H} 8$ tended towards an increase. The final and the local minima all were higher than the EMin $\mathrm{dCOM}$, which indicated the local displacement of $\mathrm{H} 3$ occurred in a direction aw ay from $\beta$-H8. (Figure S6K)

MTBD-H3 and $\beta-\mathrm{H} 9$

For $\beta-\mathrm{H} 9$, the considerable range of the percent helicities of residues in this helix should be noted, where a minimum of $29.3 \%$ was observed at Val195, and a maximum of $84.9 \%$ was observed at Tyr185. Val195 was located in the portion of $\mathrm{H} 9$ oriented the closest to MTBD-H3. Tyr185 was located on the opposite end of $\beta-\mathrm{H} 9$ from Val195, and therefore, the only possible interactions of this residue would have been within the $\beta$-tubulin. (Figure S5)

In terms of local movement of $\beta-\mathrm{H} 9$ and $\mathrm{H} 3$, much of the same trend was observed, where their distance increased to the final magnitude of $20.7 \AA$. Given the increasing distance, the displacement of the MTBD-H3 was also away from $\beta$-H9. (Figure S6L)

MTBD-H3 and $\beta-\mathrm{H} 12$ and $\beta-\mathrm{H} 18$

Given that the MTBD-H3 average helicity was 78.1\%, it appears that the entire helix remained relatively stable and folded throughout the majority of the trajectory. (Figure S5) 
For $\beta-\mathrm{H} 12$ and MTBD-H3, several peaks in the distance between the helices indicated the progression of $\beta-\mathrm{H} 12$ and $\mathrm{H} 3$ farther apart, since the distance between these segments tended towardsan increase, which showed the local displacement of MTBD-H3 aw ay from $\beta-\mathrm{H} 12$. In terms of MTBD-H3 and $\beta$ H18, the dCOM was increasing throughout the trajectory, displaying that MTBD-H3 displacement was in a direction away from $\beta-H 18$. (Figure S6M, Figure S6N)

Together, the directionality of the MTBD-H3 movement was consistent across the $\beta$-tubulin segments investigated. Local movement of MTBD-H3 occurred in a direction away from $\beta-\mathrm{H} 8, \beta-\mathrm{H} 9, \beta-\mathrm{H} 12$ and $\beta-H 18$, and simultaneously, displacement occurred in the direction of $\alpha-\mathrm{B} 5$.

\section{MTBD-H6}

The average RMSF value of MTBD-H6 (Tyr3464-Ala3470) was $1.62 \AA$ with a maximum RMSF value observed at Tyr3464 (2.04 $\AA$ ) and a minimum RMSF value observed at Ala3470 (1.27 $\AA$ ).

\section{(Figure 3)}

In terms of MTBD-H6 helicity, the lowest percent helicity was observable for Glu3465 (52.9\%), and the greatest percent helicity was observable for Thr3466 (79.3\%). Both residues were positioned on the same side of the helix (as they were neighbours), which was oriented away from the rest of the tubulin heterodimer. Neither was in close enough proximity to either tubulin chain for any interaction to occur. Both residues could only have interacted with other residues in MTBD-H6 or the MTBDH5-H6 loop. (Figure S5, Figure S7)

MTBD-H6 and $\alpha$-B5

Local displacement of MTBD-H6 was in the direction of $\alpha-\mathrm{B} 5$ as the $\mathrm{dCOM}$ between these segments decreased over the course of the trajectory. The EMin dCOMwas $38.1 \AA$, and the initial $\mathrm{dCOM}$ was $36.2 \AA$ ( $\sim 1 \mathrm{~ns})$. Both values were greater than the convergence $\mathrm{dCOM}(35.5 \AA$ at $\sim 50 \mathrm{~ns})$ and the dCOM at the final conformation (35.8 $\AA$ at $\sim 199$ ns). (Figure 6C)

\section{MTBD-H6 and $\alpha-\mathrm{H} 16$}

A hydrogen-bonding interaction was detected between Ser3471 (MTBD-H6-H7 loop) and $\alpha$ Glu415 ( $\alpha$-H16), with decreasing distance between the amino acids. (Figure S6P) Arg3469 (MTBD-H6) and $\alpha$-Gly416 (H16) also interacted through H-bond with decreasing distance $(6.13 \AA)$ compared to that in the EMin structure $(9.93 \AA)$. This positioning suggested that the conformation of the subunits was perhaps approaching an intermediate conformation that 
transitioned the system towards a lower affinity mode (closer to a $\beta$-registry status). It is proposed that a salt bridge formation between $\alpha$-Glu415 and MTBD Arg3469 may allow the formation of the CC2MT linkage, which facilitates the formation of the high-affinity conformations [6]. (Figure S6S) Arg3469 (MTBD-H6) was also found to form a salt bridge with $\alpha$-Gly414 (H15-H16 loop). (Figure S6T)

Given the proximity of these interacting pairs, it is likely that the H-bond between Arg3469 (MTBDH6) and $\alpha$-Gly416 (H16) also contributes to the formation of this linkage in some capacity. (Figure S6Q)

MTBD-H6 and $\beta-H 6-H 7$ loop

$\beta$-Glu447 and $\beta$-Glu449 (of $\beta$-CTT) interacted across the interface with Arg3469 (MTBD-H6) and Lys3472 (MTBD-H6-H7 loop) by salt-bridge formation. (Figure S6G and S6H \& Table S1) The results are in agreeance and indicate that MTBD-H6 locally shifted towards the direction of $\alpha$-B5 and $\alpha-\mathrm{H} 16$. This is an important finding considering that MTBD-H6 was a main helix at the MTBD$\alpha$-tubulin interface.

\subsection{Large Scale Collective Motions}

The collective dynamic behavior of the heterotrimer system was analyzed based on the motions of mass-weighted coordinates of all atoms obtained by means of NMA and Essential Dynamics (ED) [38, 40]. The latter, also known as Principal Component Analysis (PCA), was implemented to study the relation between large-scale collective motions of each subunit (i.e. $\alpha$ tubulin, $\beta$-tubulin or MTBD) and their functional properties (i.e. MTBD low-high affinities). The focus was on the subunit segments involved in the interfacial binding area, similar to the study of local interactions in the preceding section.

The "scree plot" showed the mean square displacements of atoms $\left(\mathrm{MSD} / \mathrm{nm}^{2}\right)$ and correlated each eigenvalue to their corresponding eigenvector, each representing one mode of the directional motion of the subunits. The eigenvalues and the concerted motions, associated with the first five eigenvectors with the largest magnitudes, were analyzed. (Figure 8A and $\mathbf{8 B}$ )

The projection of the MD trajectory onto its first and second (PC1 vs. PC2) eigenvectors showed the overlap of vector subspaces from PC1 and PC2, corresponding to the conformational ensembles of the MTBD binding to the tubulin heterodimer in a two-dimensional presentation. (Figure 8C) 
The conformational ensembles corresponding to the essential eigenmodes were screened for elucidating the structural mode of the MTBD binding affinity to the tubulin heterodimer. The RMSD, as well as the variation of the radius of gyration of the heterotrimer both showed the convergence of the system near 50 ns of the MD trajectory. The RMSD of the MTBD decreased $\sim 1.3 \AA$ around $\sim 130$ ns of trajectory, presenting a major and stable conformation of the MTBD bound to the tubulin heterodimer. (Figure S7 and Figure 8)

A

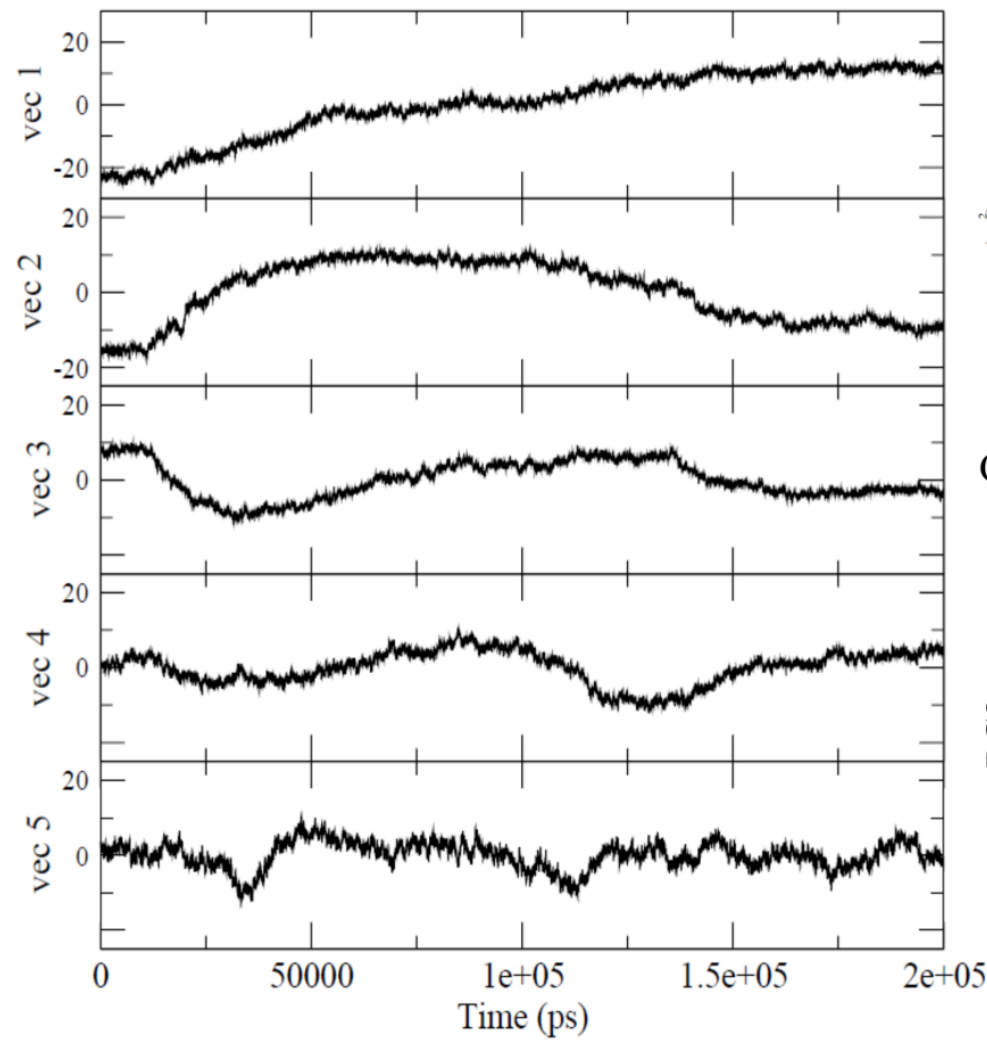

B

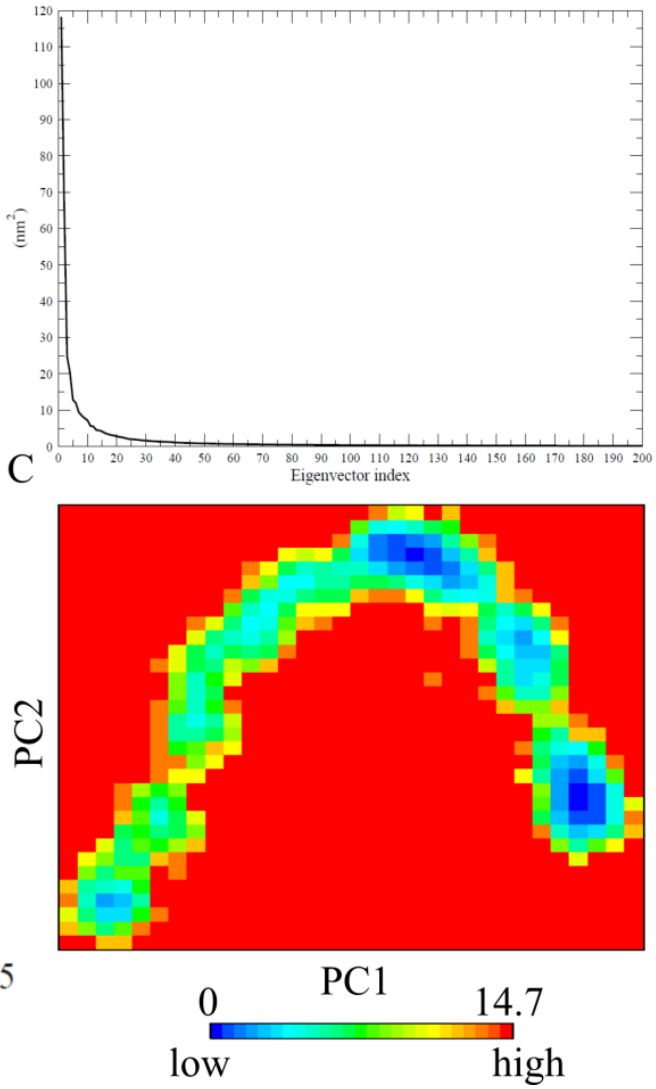

Figure 8: Principal component analysis. (A) Projection of trajectory on the first five eigenvectors, eigenvalues (nm) vs. time (ps), (B) Scree plot of eigenvalues $\left(\mathrm{nm}^{2}\right)$ vs. eigenvector index, (C) A histogram obtained from projecting the trajectory on the first eigenvector (PC1) versus the second eigenvector (PC2).

In the structure of the complex from cryo-EMic [6], the MTBD-H1 is a fragment of coiled-coil 1 (CC1), while MTBD-H6 extends to coiled-coil 2 (CC2); thus the movements of H1 and H6 against one another reflect that of CC1 and CC2, which are known to change the MTBD status between high- and low-affinity corresponding to the respective registry [13, 41]. 
In addition, the CTTs of tubulin subunits have been shown to play important roles in facilitating motor protein movement along the MT [42] and mediating the interactions with the MTassociated proteins (MAPs). Parker et al., have presented the $\beta$-CTT as a key regulator of MT dynamics, suggesting its direct involvement in MT-related cellular activities [43]. Since the tubulin CTTs are also important sites for chemical and post-translational modifications (PTM) and also the focal point for the study of the "tubulin code" [44] their structural and dynamic conformational changes necessitate close assessment. As mentioned, we used a completed structure of heterodimer tubulin according to the corresponding sequence data, which included the missing residues from the cryo-EMic and the $\mathrm{X}$-ray structure of the tubulin-MTBD. Therefore, the resulting simulation data facilitated evaluating the effects of these newly constructed segments on the conformational transformation of the MTBD potentially from low- to high-affinity.

Low-frequency motions of the system, associated with the first eigenvector, showed an orchestrated motion of a cluster of MTBD-tubulin heterodimer conformations, where the MTBD$\mathrm{H} 1$ rotated on the $\mathrm{XZ}$ surface from its initial position with respect to the axis $(\mathrm{Y}$, along the tubulin heterodimer) and resulted in observation of additional conformation to those seen in the electron microscopy [6] or crystal structure [10] of the MTBD. (Figure 9)

The formation of the distinct conformations of the MTBD helices, detected on the scree plot, was due to the effect of the $\beta$-CT'T residues (Ala438-Ala455), which were absent in the pseudo-atomic model of MTBD-tubulin EMic map (3J6P) [6].

By fitting a crystal structure of the MTBD (resolution of $2.8 \AA$ ) [14] into the EMic map, the MT-MTBD model was constructed (3J6P) by Uchimura et al., where most of the MTBD helices were just roughly fitted. In addition, a part of the density, parallel to the $\beta-\mathrm{H} 18$, was not completely filled by the structure. Uchimura et al., have indicated that the best fitting was possible by shifting the $\mathrm{H} 1$ of the MTBD in its N-terminal for filling the space [6]. Thus, it is possible that the absence of tubulin heterodimer bound to the MTBD, in the crystal structure (3VKH) [14], used for solving the EMic map, affected the resulting conformation [6].

The observed interactions of the $\beta$-CTT residues revealed their roles in shifting the MTBDH1. Coordinated movements were detected in a set of conformations from the first PCA eigenvector with the highest mean squared displacements (MSD) of $118 \mathrm{~nm}^{2}$. The $\beta$-CTT underwent high fluctuation and simultaneously interacted with MTBD-H1 and -H6 when it was in close proximity to each helix. The tail also indirectly affected movements of MTBD-H1, since $\beta-\mathrm{H} 18$ (attached to the $\beta$ CTT) and the MTBD-H1 acted together at the $\beta$-tubulin-MTBD interface. An ensemble of 
conformations exhibited the interchangeable transformations of the MTBD-tubulin conformations from a low- to high-affinity and vice versa, where the MTBD-H1 shifted to a nearly parallel position relative to the heterodimer and returned to its initial position. The binding and subsequent switch to the high-affinity ( $\alpha$-registry) should bring MTBD-H1 to a more parallel position to the MT central axis [13]. (Figure 9)

As well, both of the conformations presented coordination-changes of the MTBD-H1-H8 residues with more pronounced displacements at MTBD-H1, - H3, - $\mathrm{H} 5$ and -H7.

The MTBD-H1 of conformation 1 rotated nearly $+45^{\circ}$ on the XZ surface because of its interaction with $\beta$-H18 and the highly fluctuating $\beta$-CT'T. The $\beta$-CT'T segment also interacted with H6 and $\mathrm{H} 7$ such that its effect on the MTBD-H6 resulted in $\sim+90^{\circ}$ rotation of the former on the ZY surface, compared to their position in the EMin structure [6]. (Figure 9)

The electrostatic interactions of the tubulin CTTs have been suggested to play critical roles in tubulin structural stability and function [42] as they could affect processing electrical signals by the MTs [45]. Tubulin C-termini interact with the surface of the subunits, and given their high flexibilities, they explore interaction sites in the surrounding environment (e.g. with MAPs). However, the preferable orientations are farther away from the surface of the tubulins [46]. Another study has suggested that tubulin $\mathrm{C}$-termini may affect their conformations and consequently their functions, since the $\alpha$-CTT interacts with the $\beta$-H11, whereas $\beta$-CTT showed interactions with $\alpha$-H11 [42].

The $\beta$-H18 and especially the $\beta$-CTT were rich in negatively charged residues (i.e., Glu417 Glu420, Glu422, Glu431, Glu443, Glu448, Glu449, Glu446, Glu447, Asp427, Asp441, Asp445 and Asp453). There were also a number of residues containing an aromatic ring that contribute to the negative electrostatic property of this $\beta$-tubulin region (i.e., Phe418, Tyr432, Tyr435 and Phe446). 

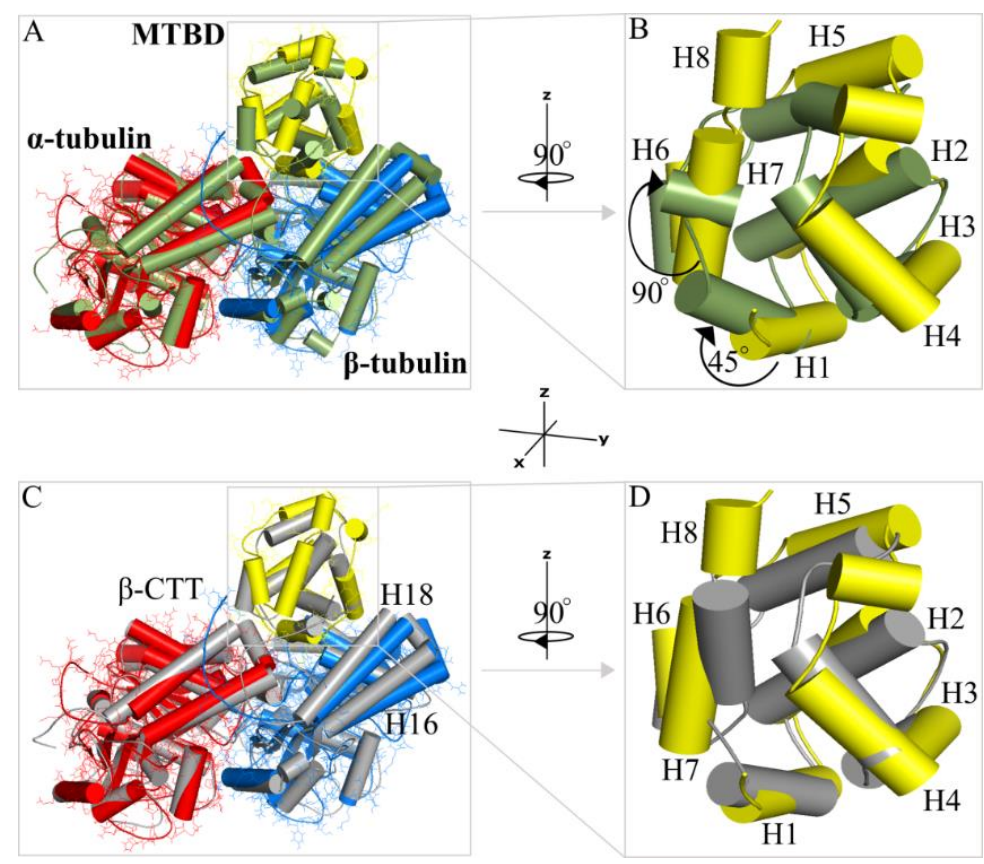

Figure 9: Superposition of the two distinct conformations of the heterotrimeric complex from the first PCA (gray and green), on the EMin structure (dynein, yellow; $\alpha$-tubulin, red; $\beta$-tubulin, blue). (A) Conformation 1 (green), (B) close view of the MTBD in conformation 1, (C) conformation 2 (gray), and (D) close view of the MTBD in conformation 2.

Thus, they create a counter electrostatic environment for the $\beta$-CTT, which is external to the tubulins. The electrostatic charge distribution on MTBD-H1, - H3, - H4, - H6 and - $\mathrm{H} 7$ that closely interacted with the $\beta$-CTT or $\beta$-H18 mainly created a positive electrostatic surface, (i.e., Lys3441, Lys3442, Lys3454, Arg3469 and Lys3472). Consequently, the $\beta$-CTT or $\beta$-H18-MTBD interactions were thought to be regulated by the electrostatic charge distributions on these segments. (Figure 10 and Figure S3) 


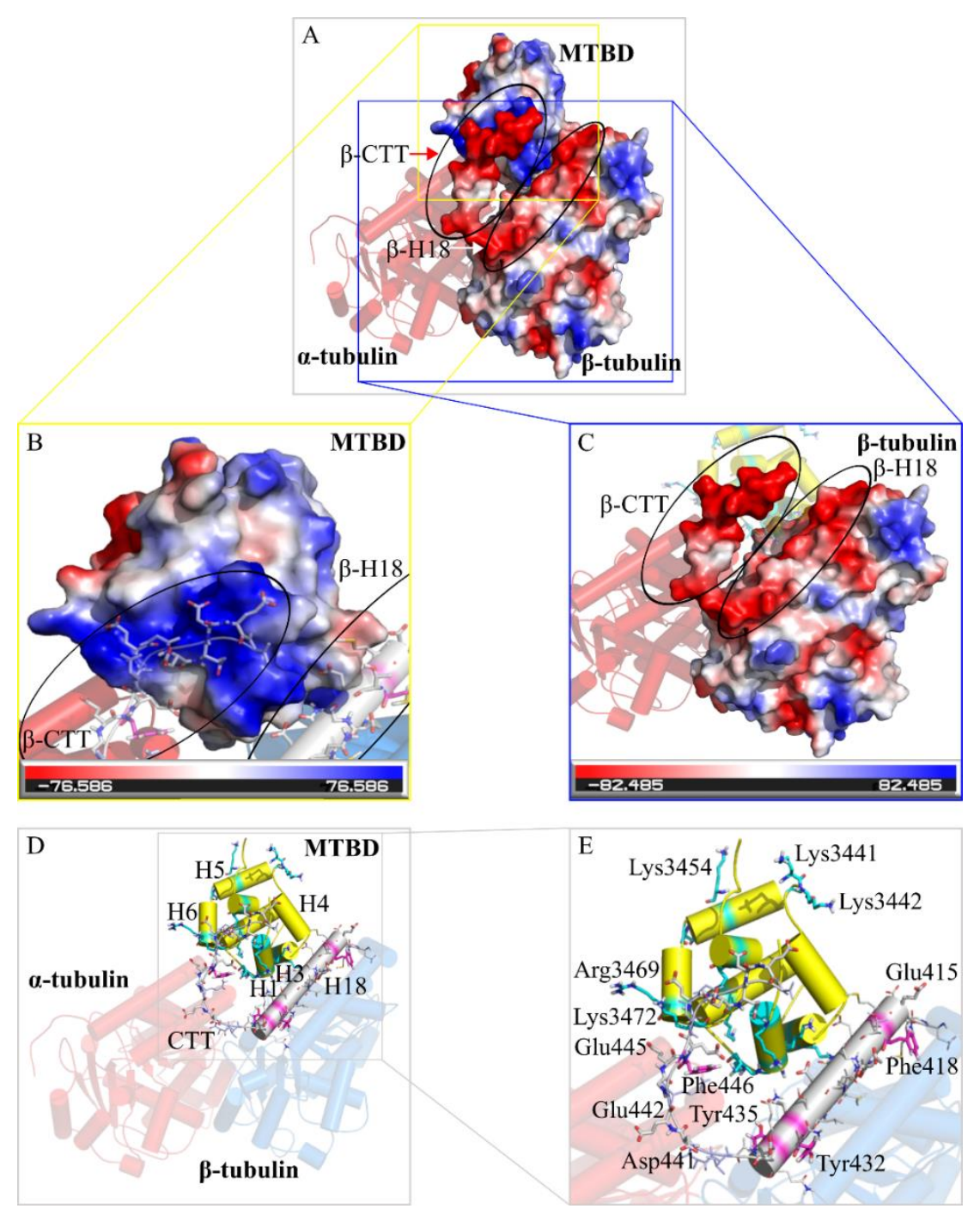

Figure 10: Electrostatic interactions between the MTBD and $\beta$-tubulin, (A) the negative electrostatic surface covering $\beta$-CTT and $\beta$-H18 (red surface) and the positive electrostatic patch on the MTBD surface (blue), a close view of (B) the positive electrostatic patch on the MTBD, (C) the negative surface on the $\beta$-CTT and $\beta$-H18, (D) the positively charged residues generating the positive electrostatic surface on the MTBD that interact with $\beta$-tubulin, (E) some of the negatively charged residues (gray stick) of the $\beta$-CTT (gray loop) and the $\beta$-H18 (gray helix) consisting of aromatic residues (magenta). For simplicity, only some of the negatively charged residues are labelled; nonlabelled residues (gray sticks) include Glu420, Glu422, Glu431, Glu443, Glu448, Glu449, Glu446, Glu447 and Asp427, Asp441, Asp445 and Asp453.

The NMA results exhibited relatively larger displacements of $\alpha$-tubulin than of the $\beta$-tubulin and the MTBD. The RMSF of each domain in the lowest frequency mode (mode 1) was relatively 
within the range of the calculated RMS for the corresponding local fluctuations. (Figure $\mathbf{1 1}$ and Figure 3)

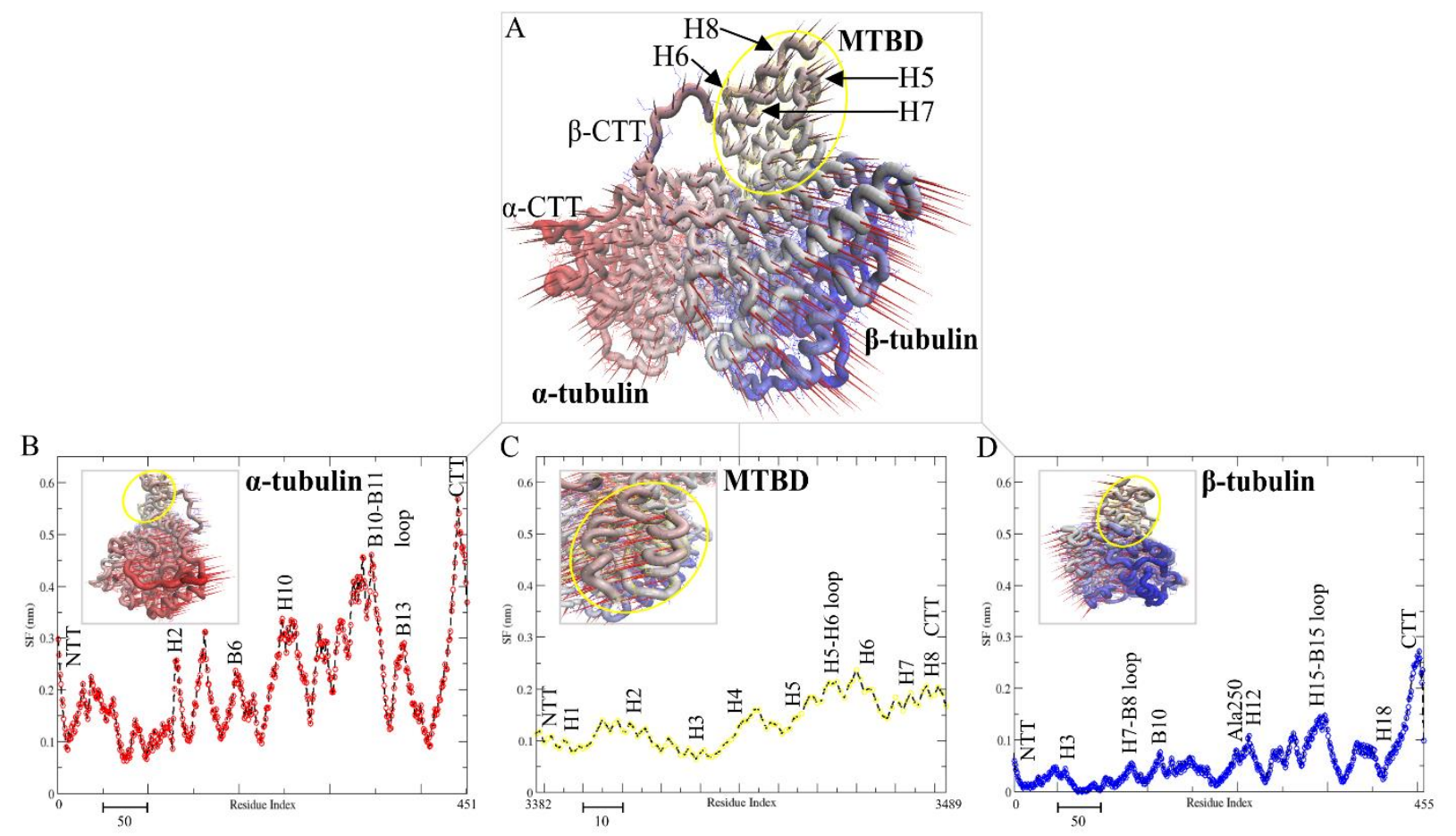

Figure 11: NMA figure showing (A) heterotrimer, Square fluctuation graph of (B) $\alpha$-tubulin, (C) MTBD, and (D) $\beta$-tubulin. Structural representations of the heterotrimer were colored based on the degree of segment movement with ranges of rigid (grey) to flexible (pink).

The $\alpha$-tubulin motions expanded from $5.7 \AA$ to $7.0 \AA$, with the largest observable displacement being related to the $\alpha$-CTT. How ever, this tail remained far away from the MTBD with minimal effect on its conformational change, in contrast to the $\beta$-CTT. The $\alpha$-NTT was highly fluctuating (up to $3.0 \AA$ ), and was the next largest RMSF peak of $\alpha$-tubulin after the $\alpha$-B10-B11 loop which fluctuated up to $4.5 \AA$. (Figure 11)

The $\beta$-tubulin motion was maximized at its CTT (2.8 $\AA$ ), followed by that of H15-B15 loop $(1.5 \AA)$, whereas $\beta$-tubulin residues from its NTT to Ala250 remained relatively rigid with RMSFs below $1.0 \AA$. Similar to the low-frequency conformations of the first eigenvector of PCA, the NMA showed that the $\beta$-CTT oscillated in close proximity to the MTBD-H6 and MTBD-H7, along which it moved in a concerted fashion with the displacement of up to $2.6 \AA$. The effects of the counter-ion amino acids discussed above facilitated the combined and co-directional movements of the MTBD helices $(\mathrm{H} 6, \mathrm{H} 7)$ and the $\beta$-CTT. The $\mathrm{H} 4$ to $\mathrm{H} 8$ of the MTBD also underwent fluctuations ranging 
from $1.0 \AA$ to $2.0 \AA$ with the highest magnitude of the RMSF at the H5-H6 loop consisting of Thr3453 to Asp3463. (Figure 11)

The RMSF obtained from the MD simulation also showed the high fluctuations of the MTBD-H1, -H5, -H4-H5 loop, as well as the MTBD-H8. (Figure 3)

\section{Conclusions}

Study of the collective motions of the MT-MTBD heterotrimeric structure with NMA and PCA assisted in the identification of conformations that could serve in the refinement of the X-ray structure and cryo-EMic map data; this allowed elucidation of the conformational changes of the domains and assessment of the important effects of the residues of the system, which were absent in the experimentally solved structures. In this work, the structural mechanism of the interactions between $\beta$-CTT and $\beta-H 18$ with the MTBD-H1 and -H6 were explained. The interaction network in the area dominated by the electrostatic forces exhibited the orchestrated movements of the helices engaging the $\beta$-CTT, which could profoundly affect the formation of the low-to-high binding modes of the MTBD with respect to $\beta$-tubulin. Analyses of the local movements through the MD simulation showed that the MTBD-H1 was oriented perpendicular to the tubulin dimer at its interface. H1 was oriented with its $\mathrm{N}$-terminal positioned in close proximity to the $\beta$-CTT. MTBD-H3 was perpendicular to the tubulin dimer with its $\mathrm{C}$-terminal raised relative to the rest of the helix. In a significant portion of the MD trajectory, the positioning of these helices was close to the low -affinity conformation. The system became progressively stable, which accounts for the conformational switch into a more stabilized form of the low-affinity conformation. Further investigation of $\mathrm{H} 1$ and $\mathrm{H} 3$ position with the PCA showed that $\mathrm{H} 1$ could rise up to $45^{\circ}$ from its initial position in the EMinstructure and cause conformational transitions. Therefore, we have also acknowledged the presence of other conformational states of the MTBD, including an increasingly stabilized low-affinity, an intermediate and high-affinity conformation with a thorough conformational sampling using the PCA.

Revision of the dynamic conformational changes of the heterotrimeric system also highlighted the structural information regarding the position and orientation of the residues, which formed salt bridges. These included the interactions between Glu3390 (MTBD-H1) and $\alpha$-Arg402 ( $\alpha$-H14-H15 loop), as well as Lys3396 (H1-H2 loop) and $\alpha$-Glu411 (H15), both of which were detected between the MTBD and $\alpha$-tubulin. During the trajectory, the dCOM values between Lys3396 (MTBD-H1-H2 loop) and $\alpha$-Glu411 ( $\alpha$-H15) fluctuated above the EMin dCOM values. This indicated movement of 
the MTBD-H1-H2 loop farther away from $\alpha-\mathrm{Glu} 411(\alpha-\mathrm{H} 15)$, which was found to form a salt bridge with Lys3402 (MTBD-H2).

The H-bond formed between Arg3469 (MTBD-H6) and $\alpha$-Gly416 (H16) became stronger as the MD simulation time progressed, which was indicated by decreasing $\mathrm{dCOM}$ values. Uchimura et al., proposed that the salt bridge formation between MTBD Arg3469 and $\alpha$-Glu415 may facilitate the formation of the high-affinity conformations, which was also exhibited by the observed conformation obtained from the MD simulation in this work. The H-bond may have an influence on the salt bridge formation between MTBD Arg3469 and $\alpha$-Glu415.

The interactions between $\alpha$-Arg402 ( $\alpha$-H14-H15 loop), with Glu3390 (MTBD-H1) and that of $\alpha$-Gly416 (H16) with Arg3469 (MTBD-H6), are thought to play an important role in allowing the registry switch that facilitates ATPase activation. Our study presented computational structural evidence that supports explaining the effective role of involved residues on the conformational transitions. Those included three particular residues from $\beta-H 18$ - Glu420, Asp427 and Glu431 which formed salt-bridges with MTBD-H1. Over half of the residues in tubulin, involved in salt bridges, were located in either $\beta$-H18 or the $\beta$-CTT. For each of these residues, at least one interaction with MTBD-H1 was found. In addition, both $\beta-$ Glu447 and $\beta$-Glu449 (of $\beta$-CTT) interacted across the interface with Arg3469 (MTBD-H6) and Lys3472 (MTBD-H6-H7 loop) demonstrating that MTBD-H6 shifted locally towards the direction of $\alpha$-tubulin during the simulation. This was an important structural observation considering that MTBD-H6 was a main helix at the MTBD- $\alpha$-tubulin interface. In addition, Lys3479 (MTBD-H7) was found to form a salt-bridge with $\beta$-Glu449, which was strengthened towards the end of the trajectory as the residues became closer than their coordination in the EMin structure.

Lys3479 (MTBD-H7) was also shown to interact with $\beta$-Glu449 of $\beta-H 18$, through salt bridge formation. The strength of this interaction was expected to improve over the trajectory as these residues became closer than in the EMin structure. Additionally, new salt bridges were observed between $\beta$-Glu447 (C'T'T) and Lys3384 (MTBD-H8), Lys3386 (MTBD-H1) and His3387 (MTBD-H1) as well as between $\beta$-Glu449 (CTT) with Lys3384 (MTBD-H8), Lys3386 and His3387 (MTBD-H1). These were obtained as a result of including the CTT in the study of the heterotrimeric structure that added up to the structural evidence that supports the importance of the tubulin terminal tails in dynein movement. A number of hydrogen bonds were also identified between the MTBD and $\beta$-tubulin; our investigation detected up to three H-bonds between MTBD and $\beta-\mathrm{H} 18$, and up to four with $\beta$-CTT.

In consideration of the presented results, it should be noted that this system included the tubulin 
heterodimer and the MTBD, while the presence of the remainder of the dynein structure in future studies would be necessary to study more complex factors affecting the directionality of dynein movement. The computational study presented here, attempted to assist in filling the knowledge gap caused by the instrumental limitations or technical difficulties in experimental structural biology while acknowledging the great value and importance of the existing experimental data in the field.

\section{Supplementary Information}

Figure S1: Amino acid sequences of (A) $\alpha$-tubulin, (B) $\beta$-tubulin and (C) MTBD retrieved from the PDB. Root Mean Square Deviation (RMSD) data from (D) 200 ns trajectory.

Figure S2: Sequence alignment data of (A) D. discoideum and M. musculus cytoplasmic dynein heavy chain, (B) S. scrofa and S. cerevisiae $\alpha$-tubulin, and (C) S. scrofa and S. cerevisiae $\beta$-tubulin. Asterisk $\left(^{*}\right)$ denotes identical amino acid for both species, while a singular dot (.) denotes amino acids of different R-chain groups and a colon (:) denotes amino acids with R-chains of the same group.

Figure S3: dCOM between (A) $\beta$-H18 and MTBD, (B) $\beta$-CTT and MTBD, (C) $\beta$-H18 and MTBDH1, and (D) $\beta$-CTT and MTBD-H1.

Figure S4: Number of hydrogen bonds between (A) MTBD and $\beta$-tubulin, (B) MTBD and $\beta$-H18 and $(\mathrm{C})$ MTBD and $\beta$-CTT

Figure S5: Percent helicity of MTBD-tubulin interface residues, (A) MTBD-H1, (Lys3385- Ser3393, 2-10), (B) MTBD-H3, (Trp3419-Ile3426, 2-9), (C) MTBD-H6, (Tyr3464-Ala3470, 2-8), (D) $\alpha-H 15$ (Val405-Glu411, 2-8), (E) ß-H8, (Met149-Glu160, 2-12), (F) $\beta-H 9$, (Glu183-Asn197, 2-16), and (G) $\beta-H 18$ (Glu415-Gln436, 2-23).

Figure S6: dCOM between (A) MTBD-H1 and -H3, (B) MTBD-H1 N-terminal and -H3 N-terminal, (C) MTBD-H1 and $\alpha$-H14, (D) MTBD-H1 and $\alpha$-H15, (E) Ser3393 (MTBD-H1) and $\beta$-Pro263, (F) $\alpha$-Glu411 (H15) and Lys3396 (black) and Lys3402 (red) in the MTBD-H1-H2 loop and MTBD-H2 respectively, (G) $\beta$-Glu447 ( $\beta$-tubulin C-terminal) and MTBD Lys3384 (black) in the N-terminal, Lys3386 (red) in H1, His3387 (blue) in H1, Arg3469 (green) in H6, and Lys3472 (orange) in the H6-H7 loop, $(\mathrm{H}) \beta$-Glu449 ( $\beta$-tubulin C-terminal) and MTBD Lys3384 (black) in the N-terminal, Lys3386 (red) in H1, His3387 (blue) in H1, Arg3469 (green) in H6, Lys3472 (orange) in 
the H6-H7 loop and Lys3479 (magenta) in H7, (I) MTBD-H2 and $\alpha-H 15$, (J) Thr3399 (MTBD-H2) and $\alpha$-Glu414 (H15-H16 loop), (K) MTBD-H3 and $\beta-H 8$, (L) MTBD-H3 and $\beta-H 9$, (M) MTBD-H3 and $\beta-H 12$, (N) MTBD-H3 and $\beta-H 18,(\mathrm{O})$ MTBD-H6 and $\alpha-H 16$, (P) Ser3471 (MTBD-H6-H7 loop) and $\alpha$-Glu415 (H16), and (Q) $\alpha$-Gly416 (H16) and Arg3469 (MTBD H6), (R) Oxygen atoms (OE1-9186 and OE2-9187) of the Glu3390 (MTBD-H1) carboxyl group and the nitrogen atoms (NE-4004, NH1-4007 and NH2-4010) of the $\alpha$-Arg402 (H14-H15 loop) amine group, (S) Oxygen atoms (OE1-4152 and OE2-4153) of the $\alpha$-Glu415 (H16) carboxyl group and the nitrogen atoms (NE-9977, NH1-9980 and NH2-9983) of the Arg3469 (MTBD-H6) amine group, (T) Oxygen atoms (OE1-4142 and OE2-4143) of the $\alpha$-Glu414 (H15-H16 loop) carboxyl group and the nitrogen atoms (NE-9977, NH1-9980 and NH2-9983) of the Arg3469 (MTBD-H6) amine group, and (U) oxygen atoms (OE1-4152 and OE2-4153) of the $\alpha$-Glu415 (H16) carboxyl group and the nitrogen atoms (NE-4004, NH1-4007 and NH2-4010) of the $\alpha$-Arg402 (H14-H15 loop) amine group.

Figure S7: (A) Radius of gyration $\left(\mathrm{R}_{\mathrm{g}} / \mathrm{nm}\right)$ vs. time (ps), (B) Root mean square deviation (nm) Figure S8: Sample of conformations presenting the fluctuation of $\beta$-CT'T.

Table S1: Salt bridge formation across the MT-MTBD interface. Interactions were identified when the distance between oxygen atoms in the carboxyl group (acidic residue side chain) and nitrogen atoms in the amine group (basic residue side chain) fell within $3.2 \AA$ cut-off at least once during 200 ns simulation.

\section{Acknowledgement}

The authors acknowledge funding supports from the Natural Sciences and Engineering Research Council of Canada (NSERC), Discovery Grant (No. 212654), awarded to L.A.; and NSERC Student Research Scholarship (No. 539417), awarded to K.H.

The authors thank Compute Canada, for accessing their HPC facilities free of charge, and the technical supports by the technical staff of ACNET and WestGrid.

\section{Associated Content}

The atomic coordinates and experimental data will be available upon reasonable request. 


\section{References}

1. Reck-Peterson SL, Redwine WB, Vale RD, Carter AP. The cytoplasmic dynein transport machinery and its many cargoes. Nat Rev Mol Cell Biol. 2018;19(6):382-98. Epub 2018/04/18. doi: 10.1038/s41580-018-0004-3. PubMed PMID: 29662141.

2. Nogales E. Structural Insights into Microtubule Function. Annual Review of Biochemistry. 2000;69(1):277-302. doi: 10.1146/annurev.biochem.69.1.277. PubMed PMID: 10966460.

3. Alisaraie L, Tuszynski JA. Determination of Noscapine's Localization and Interaction with the Tubulin- $\alpha / \beta$ Heterodimer. Chemical Biology \& Drug Design. 2011;78(4):535-46. doi: 10.1111/j.1747-0285.2011.01189.x.

4. Li Z, Alisaraie L. Microtubules dual chemo and thermo-responsive depolymerization. Proteins. 2015;83(5):970-81. doi: 10.1002/prot.24793.

5. Tuszynski J, Craddock TA, Mane J, Barakat K, Tseng C-Y, Gajewski M, et al. Modeling the Yew Tree Tubulin and a Comparison of its Interaction with Paclitaxel to Human Tubulin. Pharmaceutical Research. 2012;29(11):3007-21. doi: 10.1007/s11095-012-0829-y. 6. Uchimura S, Fujii T, Takazaki H, Ayukawa R, Nishikawa Y, Minoura I, et al. A flipped ion pair at the dynein-microtubule interface is critical for dynein motility and ATPase activation. J Cell Biol. 2015;208(2):211-22. Epub 2015/01/15. doi: 10.1083/jcb.201407039. PubMed PMID: $25583999 ;$ PubMed Central PMCID: PMCPMC4298687.

7. Kolomeisky AB. Motor proteins and molecular motors: how to operate machines at the nanoscale. J Phys Condens Matter. 2013;25(46):463101. doi: 10.1088/0953-8984/25/46/463101. PubMed PMID: 24100357; PubMed Central PMCID: PMCPMC3858839.

8. Hirakawa E, Higuchi H, Toyoshima YY. Processive movement of single 22S dynein molecules occurs only at low ATP concentrations. Proceedings of the National Academy of Sciences. 2000;97(6):2533. doi: 10.1073/pnas.050585297.

9. Shingyoji C, Higuchi H, Yoshimura M, Katayama E, Yanagida T. Dynein arms are oscillating force generators. Nature. 1998;393(6686):711-4. doi: 10.1038/31520.

10. Carter AP, Garbarino JE, Wilson-Kubalek EM, Shipley WE, Cho C, Milligan RA, et al. Structure and functional role of dynein's microtubule-binding domain. Science. 2008;322(5908):1691-5. Epub 2008/12/17. doi: 10.1126/science.1164424. PubMed PMID: 19074350; PubMed Central PMCID: PMCPMC2663340.

11. Rank KC, Rayment I. Functional asymmetry in kinesin and dynein dimers. Biol Cell. 2013;105(1):1-13. Epub 2012/10/17. doi: 10.1111/boc.201200044. PubMed PMID: 23066835; 
PubMed Central PMCID: PMCPMC3535566.

12. Hirokawa N, Niwa S, Tanaka Y. Molecular motors in neurons: transport mechanisms and roles in brain function, development, and disease. Neuron. 2010;68(4):610-38. Epub 2010/11/26. doi: 10.1016/j.neuron.2010.09.039. PubMed PMID: 21092854.

13. Redwine WB, Hernandez-Lopez R, Zou S, Huang J, Reck-Peterson SL, Leschziner AE. Structural basis for microtubule binding and release by dynein. Science. 2012;337(6101):1532-6. Epub 2012/09/22. doi: 10.1126/science.1224151. PubMed PMID: 22997337; PubMed Central PMCID: PMCPMC3919166.

14. Kon T, Oyama T, Shimo-Kon R, Imamula K, Shima T, Sutoh K, et al. The $2.8 \AA$ crystal structure of the dynein motor domain. Nature. 2012;484:345. doi: 10.1038/nature10955 https://www.nature.com/articles/ nature10955\#supplementary-information.

15. Tajielyato N, Li L, Peng Y, Alper J, Alexov E. E-hooks provide guidance and a soft landing for the microtubule binding domain of dynein. Scientific Reports. 2018;8(1):13266. doi: 10.1038/s41598-018-31480-9.

16. Ravelli RBG, Gigant B, Curmi PA, Jourdain I, Lachkar S, Sobel A, et al. Insight into tubulin regulation from a complex with colchicine and a stathmin-like domain. Nature. 2004;428(6979):198202. doi: $10.1038 /$ nature02393.

17. Löwe J, Li H, Downing KH, Nogales E. Refined structure of $\alpha \beta$-tubulin at $3.5 \AA$ resolution11Edited by I. A. Wilson. Journal of Molecular Biology. 2001;313(5):1045-57. doi: https://doi.org/10.1006/jmbi.2001.5077.

18. Alisaraie L, Rouiller I. Full-length structural model of RET3 and SEC21 in COPI: Identification of binding sites on the appendage for accessory protein recruitment motifs. Journal of molecular modeling. 2012;18:3199-212. doi: 10.1007/s00894-011-1324-9.

19. Kelley LA, Sternberg MJE. Protein structure prediction on the Web: a case study using the Phyre server. Nat Protocols. 2009;4(3):363-71.

20. Chenna R, Sugawara H, Koike T, Lopez R, Gibson TJ, Higgins DG, et al. Multiple sequence alignment with the Clustal series of programs. Nucleic Acids Research. 2003;31(13):3497-500. doi: $10.1093 /$ nar/gkg500.

21. Raghava GP, Searle SM, Audley PC, Barber JD, Barton GJ. OXBench: a benchmark for evaluation of protein multiple sequence alignment accuracy. BMC Bioinformatics. 2003;4:47. doi: 10.1186/1471-2105-4-47. PubMed PMID: 14552658; PubMed Central PMCID: PMCPMC280650. 22. Koonce MP, Grissom PM, McIntosh JR. Dynein from Dictyostelium: primary structure 
comparisons between a cytoplasmic motor enzyme and flagellar dynein. Journal of Cell Biology. 1992;119(6):1597-604. doi: 10.1083/jcb.119.6.1597.

23. Ponstingl H, Krauhs E, Little M, Kempf T. Complete amino acid sequence of alpha-tubulin from porcine brain. Proc Natl Acad Sci U S A. 1981;78(5):2757-61. doi: 10.1073/pnas.78.5.2757. PubMed PMID: 7019911.

24. Krauhs E, Little M, Kempf T, Hofer-Warbinek R, Ade W, Ponstingl H. Complete amino acid sequence of beta-tubulin from porcine brain. Proceedings of the National Academy of Sciences. 1981;78(7):4156-60. doi: 10.1073/pnas.78.7.4156.

25. Schatz PJ, Pillus L, Grisafi P, Solomon F, Botstein D. Two functional alpha-tubulin genes of the yeast Saccharomyces cerevisiae encode divergent proteins. Mol Cell Biol. 1986;6(11):3711-21. doi: 10.1128/mcb.6.11.3711. PubMed PMID: 3025610.

26. Neff NF, Thomas JH, Grisafi P, Botstein D. Isolation of the $\beta$-tubulin gene from yeast and demonstration of its essential function in vivo. Cell. 1983;33(1):211-9. doi: doi.org/10.1016/00928674(83)90350-1.

27. Schmid N, Eichenberger AP, Choutko A, Riniker S, Winger M, Mark AE, et al. Definition and testing of the GROMOS force-field versions 54A7 and 54B7. Eur Biophys J. 2011;40(7):843-56. Epub 2011/05/03. doi: 10.1007/s00249-011-0700-9. PubMed PMID: 21533652.

28. Guvench O D, A M. Comparison of Protein Force Fields for Molecular Dynamics Simulations. Molecular Modeling of Proteins 2008:63-88. doi: doi.org/10.1007/978-1-59745-177$2 \_4$.

29. Van Der Spoel D, Lindahl E, Hess B, Groenhof G, Mark AE, Berendsen HJ. GROMACS: fast, flexible, and free. J Comput Chem. 2005;26(16):1701-18. Epub 2005/10/08. doi: 10.1002/jcc.20291. PubMed PMID: 16211538.

30. Hess B, Kutzner C, van der Spoel D, Lindahl E. GROMACS 4: Algorithms for Highly Efficient, Load-Balanced, and Scalable Molecular Simulation. J Chem Theory Comput. 2008;4(3):435-47. Epub 2008/03/01. doi: 10.1021/ct700301q. PubMed PMID: 26620784.

31. M.J. Abraham DvdS, E. Lindahl, B. Hess, and the GROMACS development team. GROMACS User Manual version 20182018. Available from: www. gromacs.org. 32. Leach AR. Molecular Modelling Principles and Applications. Book, Pearson Prentice Hall. 2001.

33. Essmann U, Perera L, Berkowitz M, Darden T, Lee H, Pedersen L. A smooth particle mesh Ewald method Journal of Chemical Physics. 1995;103(19):17. doi: 10.1063/1. 
34. Hess B, Bekker H, Berendsen HJC, Fraaije JGEM. LINCS: A linear constraint solver for molecular simulations. Journal of Computational Chemistry. 1997;18(12):1463-72. PubMed PMID: WOS:A1997XT81100004.

35. Bussi G, Donadio D, Parrinello M. Canonical sampling through velocity rescaling. J Chem Phys. 2007;126(1):014101. Epub 2007/01/11. doi: 10.1063/1.2408420. PubMed PMID: 17212484.

36. Bahar I, Lezon TR, Bakan A, Shrivastava IH. Normal Mode Analysis of Biomolecular Structures: Functional Mechanisms of Membrane Proteins. Chemical Reviews. 2010;110(3):1463-97. doi: $10.1021 / \operatorname{cr} 900095$ e.

37. Tozer T, Heale K, Manto Chagas C, de Barros ALB, Alisaraie L. Interdomain twists of human thymidine phosphorylase and its active-inactive conformations: Binding of 5-FU and its analogues to human thymidine phosphorylase versus dihydropyrimidine dehydrogenase. Chemical Biology \& Drug Design. 2019;94(5):1956-72. doi: 10.1111/cbdd.13596.

38. Amadei A, Linssen ABM, Berendsen HJC. Essential dynamics of proteins. Proteins: Structure, Function, and Bioinformatics. 1993;17(4):412-25. doi: 10.1002/prot.340170408.

39. Balsera MA, Wriggers W, Oono Y, Schulten K. Principal Component Analysis and Long Time Protein Dynamics. The Journal of Physical Chemistry. 1996;100(7):2567-72. doi: $10.1021 /$ jp 9536920.

40. Berendsen HJC, Hayward S. Collective protein dynamics in relation to function. Current Opinion in Structural Biology. 2000;10(2):165-9. doi: https:/ / doi.org/10.1016/S0959440X(00)00061-0.

41. Gibbons IR, Garbarino JE, Tan CE, Reck-Peterson SL, Vale RD, Carter AP. The affinity of the dynein microtubule-binding domain is modulated by the conformation of its coiled-coil stalk. The Journal of biological chemistry. 2005;280(25):23960-5. Epub 04/11. doi: 10.1074/jbc.M501636200. PubMed PMID: 15826937.

42. Freedman H, Luchko T, Luduena RF, Tuszynski JA. Molecular dynamics modeling of tubulin C-terminal tail interactions with the microtubule surface. Proteins: Structure, Function, and Bioinformatics. 2011;79(10):2968-82. doi: 10.1002/prot.23155.

43. Parker AL, Teo WS, Pandzic E, Vicente JJ, McCarroll JA, Wordeman L, et al. $\beta$-Tubulin carboxy-terminal tails exhibit isotype-specific effects on microtubule dynamics in human gene-edited cells. Life Science Alliance. 2018;1(2):e201800059. doi: 10.26508/1sa.201800059.

44. Gadadhar S, Bodakuntla S, Natarajan K, Janke C. The tubulin code at a glance. Journal of Cell Science. 2017;130(8):1347-53. doi: 10.1242/jcs.199471. 
45. Craddock TJA, Tuszynski JA, Priel A, Freedman H. Mirotubule Ionic Conduction and its Implications for Cognitive Functions. Journal of Integrative Neuroscience. 2010;09(02):103-22. doi: 10.1142/s0219635210002421.

46. Priel A, Tuszynski JA, Woolf NJ. Transitions in microtubule C-termini conformations as a possible dendritic signaling phenomenon. European Biophysics Journal. 2005;35(1):40. doi: 10.1007/s00249-005-0003-0. 